\title{
ON REPRESENTATION VARIETIES OF 3-MANIFOLD GROUPS
}

\author{
MICHAEL KAPOVICH, JOHN J. MILLSON
}

\begin{abstract}
We prove universality theorems ("Murphy's Laws") for representation varieties of fundamental groups of closed 3-dimensional manifolds. We show that germs of $S L(2)$-representation schemes of such groups are essentially the same as germs of schemes of finite type over $\mathbb{Q}$.
\end{abstract}

\section{INTRODUCTION}

In this paper we will prove that there are no "local" restrictions on geometry of representation schemes of 3-manifold groups to $P O(3)$ and $S L(2)$. Note that both groups $H=P O(3)$ and $H=S L(2)$ are affine algebraic group schemes defined over $\mathbb{Q}$, thus, for every finitely-generated group $\Gamma$, the representation schemes

$$
\operatorname{Hom}(\Gamma, H)
$$

and character schemes

$$
X(\Gamma, H)=\operatorname{Hom}(\Gamma, H) / / H
$$

are affine algebraic schemes over $\mathbb{Q}$. Our goal is to show that, to some extent, these are the only restrictions on local geometry of the representation and character schemes of fundamental groups of closed 3-manifolds. The universality theorem we thus obtain is one in many universality theorems about moduli spaces of geometric objects, see [1], [15], [5], [6], [7], [18], [13], [14].

Below is the precise formulation of our universality theorem. In what follows we will be using the notation $G=P O(3), \tilde{G}=\operatorname{Spin}(3)$.

Theorem 1.1. Let $X \subset \mathbb{C}^{N}$ be an affine algebraic scheme over $\mathbb{Q}$ and let $x \in X$ be a rational point. Then there exist:

1. An open subscheme $X^{\prime} \subset X$ containing $x$.

2. A closed 3-dimensional manifold $M$ with the fundamental group $\pi$.

3. A representation $\rho_{0}: \pi \rightarrow P O(3, \mathbb{R})$, such that the image of $\rho_{0}$ is dense in $P O(3, \mathbb{R})$.

4. An open $G$-invariant subscheme $R^{\prime} \subset \operatorname{Hom}(\pi, G)$ containing $\rho_{0}$ and a closed subscheme $R_{c}^{\prime} \subset R^{\prime}$ which is a cross-section for the action

$$
G \times R^{\prime} \rightarrow R^{\prime} .
$$

5. An isomorphism of schemes over $\mathbb{Q}$ :

$$
f: R^{\prime} \rightarrow G^{k} \times X^{\prime}, \quad f\left(\rho_{0}\right)=(1, x),
$$

for some $k$.

Remark 1.2. One can show that the same theorem holds for a homomorphism $\rho_{0}$ whose image is a finite group with trivial centralizer in $P O(3, \mathbb{R})$.

Date: September 19, 2018. 
Since the groups $P S L(2, \mathbb{C})$ and $P O(3, \mathbb{C})=G(\mathbb{C})$ are isomorphic, and

$$
\tilde{G}(\mathbb{C})=\operatorname{Spin}(3, \mathbb{C}) \cong S L(2, \mathbb{C}),
$$

"universality" for $P O(3)$-representations leads to the one for $S L(2)$-representations:

Corollary 1.3. Let $X \subset \mathbb{C}^{N}$ be an affine algebraic scheme over $\mathbb{Q}$ and $x \in X$ be a rational point. Then there exist:

1. An open subscheme $X^{\prime} \subset X$ containing $x$.

2. A closed 3-dimensional manifold $M$ with the fundamental group $\pi$.

3. A representation $\tilde{\rho}_{0}: \pi \rightarrow S U(2)<S L(2, \mathbb{C})$, such that the image of $\tilde{\rho}_{0}$ is dense in $S U(2)$.

4. An open $G$-invariant subscheme $\widehat{S} \subset \operatorname{Hom}(\pi, S L(2))$ containing $\tilde{\rho}_{0}$, such that every complex point of $\widehat{S}$ is a Zariski dense representation to $S L(2, \mathbb{C})$.

5. A regular étale covering of schemes over $\mathbb{C}$ :

$$
\tilde{f}: \widehat{S} \rightarrow \tilde{G}^{k} \times X^{\prime}, \quad \tilde{f}\left(\tilde{\rho}_{0}\right)=(1, x),
$$

with the deck-transformation group isomorphic to $\mathbb{Z}_{2}^{r}$, for some $k$ and $r$.

In particular, $\tilde{f}$ yields an isomorphism of the analytic germs

$$
\left(\operatorname{Hom}(\pi, S L(2)), \tilde{\rho}_{0}\right) \rightarrow\left(\mathbb{C}^{3 k+3} \times X^{\prime}, 0 \times x\right),
$$

for some $k \geq 1$. Thus, if the scheme $X^{\prime}$ is non-reduced at $x$, so is $\operatorname{Hom}(\pi, S L(2))$.

Remark 1.4. Despite of our efforts, we were unable to replace an étale covering with an isomorphism in Corollary 1.3. This is strangely reminiscent of the finite abelian coverings appearing in our universality theorem for planar linkages, [7]. Note that a relation between universality theorems for projective arrangements and spherical linkages was established in [6], where a finite abelian covering appeared for essentially the same reason as in the present paper.

We will see that the action of $S L(2)$ on $\tilde{R}^{\prime}$ factors through the group $P S L(2)$, which admits a cross-section. In particular, we obtain

Corollary 1.5. There exists an isomorphism of analytic germs

$$
\left(X(\pi, S L(2)),\left[\tilde{\rho}_{0}\right]\right) \rightarrow\left(\mathbb{C}^{3 k} \times X^{\prime}, 0 \times x\right) .
$$

Example 1.6. Pick a natural number $\ell$. Then there exists a closed 3-dimensional manifold $M$, an integer $n$ and a representation $\rho: \pi_{1}(M) \rightarrow S U(2)$ with dense image, so that the completed local ring of the germ

$$
\left.X\left(\pi_{1}(M), S L(2)\right),[\rho]\right)
$$

is isomorphic to the completion of the ring

$$
\mathbb{C}\left[t, t_{1}, \ldots, t_{3 k}\right] /\left(t^{\ell}\right) .
$$

This shows that the representation and character schemes of 3-manifold groups can be nonreduced (at points of Zariski density), which is why we refrain from referring to these schemes as "varieties," as it is commonly done in the literature. 
Remark 1.7. Recently Igor Rapinchuk proved a universality theorem for character schemes of groups $\Gamma$ satisfying Kazhdan's Property T: It involves representations of $\Gamma$ 's into $S L(n, \mathbb{C})$, [14, Theorem 3]. Unlike [5] and this paper, Rapinchuk's theorem applies to the entire character variety $X^{r e d}(\Gamma, S L(n, \mathbb{C}))$ minus the trivial representation (which is an isolated point). In Rapinchuk's theorem, the number $n$ (and the group $\Gamma$ ) depend on the given affine variety $X$ over $\mathbb{Q}$.

Acknowledgments. Partial financial support to the first author was provided by the NSF grant DMS-12-05312 and to the second author by the NSF grant DMS-12-06999. We are grateful to the referee for useful remarks.

\section{Preliminaries}

2.1. Representation and character schemes. We will say that a subscheme $Y \subset$ $X$ is clopen if it is both closed and open. We will use the topologist's notation:

$$
\mathbb{Z}_{m}:=\mathbb{Z} / m \mathbb{Z}
$$

is the cyclic group of order $m$.

Let $G$ be an algebraic group scheme over a field $\mathbf{k}$ of characteristic zero (this will be the default assumption through the rest of the paper) with the Lie algebra $\mathfrak{g}$. Let $\Gamma$ be a finitely-presented group with the presentation

$$
\left\langle s_{1}, \ldots, s_{p} \mid r_{1}=1, \ldots, r_{q}=1\right\rangle .
$$

(In fact, one needs $\Gamma$ only to be finitely-generated, but all finitely-generated groups in this paper will be also finitely-presented.) Every word $w$ in the generators $s_{i}, s_{i}^{-1}, i=$ $1, \ldots, p$, defines a morphism

$$
w: G^{p} \rightarrow G,
$$

obtained by substituting elements $g_{1}^{ \pm 1}, \ldots, g_{p}^{ \pm 1} \in G$ in the word $w$ for the letters $s_{1}^{ \pm 1}, \ldots, s_{p}^{ \pm 1}$. We then obtain the representation scheme

$$
\operatorname{Hom}(\Gamma, G)=\left\{\left(g_{1}, \ldots, g_{p}\right) \in G^{p}: r_{j}\left(g_{1}, \ldots, g_{p}\right)=1, j=1, \ldots, q\right\},
$$

as every homomorphism $\Gamma \rightarrow G$ is determined by its values on the generators of $\Gamma$. We will, thus, think of points of this scheme as homomorphisms $\rho: \Gamma \rightarrow G$. The representation scheme is known to be independent of the presentation of the group $\Gamma$. We refer the reader to [9, 17] for more details. We also refer the reader to [16, 17] for detailed discussion of character varieties/schemes and survey of their applications to 3-dimensional topology.

We will frequently use the following two facts about representation schemes, see e.g [17]:

1. $\operatorname{Hom}\left(\Gamma_{1} \star \ldots \star \Gamma_{k}, G\right) \cong \prod_{i=1}^{k} \operatorname{Hom}\left(\Gamma_{i}, G\right)$.

2. For each $\rho \in \operatorname{Hom}(\Gamma, G(\mathbf{k}))$ satisfying $\mathrm{H}^{1}\left(\Gamma, \mathfrak{g}_{\text {Ad } \rho}\right)=0$,

$$
\rho \in \operatorname{Hom}(\Gamma, G(\mathbf{k}))
$$

is a smooth point of the scheme $\operatorname{Hom}(\Gamma, G)$. The $G$-orbit through $\rho$ is open in $\operatorname{Hom}(\Gamma, G)$.

In what follows we will use the simplified notation $\mathrm{H}^{q}(\Gamma, A d \rho)$ instead of $\mathrm{H}^{q}\left(\Gamma, \mathfrak{g}_{A d \rho}\right)$. 
We assume from now on that the group $G$ is affine; in particular, $\operatorname{Hom}(\Gamma, G)$ is also an affine scheme. The group $G$ acts naturally on this scheme:

$$
(g, \rho) \mapsto \rho^{g}, \quad \rho^{g}(\gamma)=g \rho(\gamma) g^{-1} .
$$

Assuming, in addition, that $G$ is reductive, we obtain the GIT quotient

$$
X(\Gamma, G)=\operatorname{Hom}(\Gamma, G) / / G,
$$

which is a scheme of finite type known as the character scheme (or, more commonly, as the character variety). However, as we will see, both representation and character schemes can be nonreduced, so we will avoid the traditional representation/character variety terminology.

We will use the notation

$$
\operatorname{Hom}^{\text {red }}(\Gamma, G), \quad X^{r e d}(\Gamma, G)
$$

to denote the varieties which are the reductions of the schemes

$$
\operatorname{Hom}(\Gamma, G), \quad X(\Gamma, G) .
$$

Recall that for every $\rho \in \operatorname{Hom}(\Gamma, G)$, the vector space of cocycles

$$
Z^{1}(\Gamma, A d \rho)
$$

is isomorphic to the Zariski tangent space $T_{\rho} \operatorname{Hom}(\Gamma, G)$ and this isomorphism carries the subspace of coboundaries $B^{1}(\Gamma, A d \rho)$ to the tangent space of the $G$-orbit through $\rho$. Note, however, that $\mathrm{H}^{1}(\Gamma, A d \rho)$ is not always isomorphic to the Zariski tangent space of $[\rho] \in X(\Gamma, G)$, see $[2, \S 6]$ as well as [17].

Suppose now that the group $\Phi$ is finite. Then for every $\rho \in \operatorname{Hom}(\Phi, G)$,

$$
\mathrm{H}^{1}(\Phi, A d \rho)=0 \text {. }
$$

(Furthermore, $\mathrm{H}^{i}(\Phi, A d \rho)=0, i \geq 1$.) In particular, the $G$-orbit of $\rho$ is a clopen (closed and open) subscheme

$$
\operatorname{Hom}_{\rho}(\Phi, G) \subset \operatorname{Hom}(\Phi, G) .
$$

This subcheme is isomorphic to the quotient $G / \zeta_{G}(\rho(\Phi))$, where $\zeta_{G}(H)$ denotes the centralizer of the subgroup $H$ in $G$. (Note that if $\zeta_{G}(\rho(\Phi)$ ) equals the center of $G$, then the point $[\rho] \in X(\Phi, G)$ is a reduced isolated point in the character scheme and the entire character scheme is smooth.) We obtain:

Lemma 2.1. For every finite group $\Phi$ and connected affine group $G$, the scheme $\operatorname{Hom}(\Phi, G)$ is smooth and each irreducible component of $\operatorname{Hom}(\Phi, G)$ is $G$-homogeneous. These irreducible components are the open subschemes $\operatorname{Hom}_{\rho}(\Phi, G)$. If the representation $\rho$ is trivial, then $\operatorname{Hom}_{\rho}(\Phi, G)$ is a single point.

The following lemma is also immediate:

Lemma 2.2. Let $\phi: \Gamma \rightarrow \Gamma^{\prime}$ be a group homomorphism. Then the pull-back map $\phi^{*}(\rho)=\rho \circ \phi$ is a morphism of schemes

$$
\operatorname{Hom}\left(\Gamma^{\prime}, G\right) \rightarrow \operatorname{Hom}(\Gamma, G) .
$$


Lemma 2.3. Let $\Gamma$ be a finitely-presented group and let $\Theta \subset \Gamma$ be a finite subset with the quotient group

$$
\Gamma^{\prime}:=\Gamma /\langle\langle\Theta\rangle\rangle
$$

Let $\phi: \Gamma \rightarrow \Gamma^{\prime}$ denote the projection homomorphism. Then the pull-back morphism

$$
\phi^{*}: \operatorname{Hom}\left(\Gamma^{\prime}, G\right) \rightarrow \operatorname{Hom}_{\Theta}(\Gamma, G)
$$

is an isomorphism, where

$$
\operatorname{Hom}_{\Theta}(\Gamma, G) \subset \operatorname{Hom}(\Gamma, G)
$$

is the closed subscheme defined by

$$
\operatorname{Hom}_{\Theta}(\Gamma, G)=\{\rho \in \operatorname{Hom}(\Gamma, G): \rho(\theta)=1, \forall \theta \in \Theta\} .
$$

Proof. Given a finite presentation $P$ of $\Gamma$ let $P^{\prime}$ be the presentation of $\Gamma^{\prime}$ obtained from $P$ by adding words representing elements of $\Theta$ as the relators. Then the assertion follows immediately from the definition of the representation scheme of a group using a group presentation.

Corollary 2.4. Suppose that every element $\theta \in \Theta$ has finite order. Then the isomorphism $\phi_{*}: \operatorname{Hom}\left(\Gamma^{\prime}, G\right) \rightarrow \operatorname{Hom}_{\Theta}(\Gamma, G)$ sends $\operatorname{Hom}\left(\Gamma^{\prime}, G\right)$ to the open subscheme $\operatorname{Hom}_{\Theta}(\Gamma, G) \subset \operatorname{Hom}(\Gamma, G)$.

Proof. Consider an element $\theta \in \Theta$ and the trivial representation $\rho_{0, \theta}:\langle\theta\rangle \rightarrow G$. By Lemma 2.1, the singleton

$$
\left\{\rho_{0, \theta}\right\}=\operatorname{Hom}_{\rho_{0}, \theta}(\langle\theta\rangle, G) \subset \operatorname{Hom}(\langle\theta\rangle, G)
$$

is a reduced isolated point in the scheme $\operatorname{Hom}(\langle\theta\rangle, G)$. In particular, this point is open in $\operatorname{Hom}(\langle\theta\rangle, G)$. We have the pull-back morphism

$$
\phi_{\theta}^{*}: \operatorname{Hom}(\Gamma, G) \rightarrow \operatorname{Hom}(\langle\theta\rangle, G),
$$

induced by the inclusion homomorphism $\phi_{\theta}:\langle\theta\rangle \hookrightarrow \Gamma$. Therefore,

$$
\left(\phi_{\theta}^{*}\right)^{-1}\left(\operatorname{Hom}_{\rho_{0}, \theta}(\langle\theta\rangle, G)\right) \subset \operatorname{Hom}(\Gamma, G)
$$

is an open subscheme. Furthermore, by the definition of $\operatorname{Hom}_{\Theta}(\Gamma, G)$,

$$
\operatorname{Hom}_{\Theta}(\Gamma, G)=\bigcap_{\theta \in \Theta}\left(\phi_{\theta}^{*}\right)^{-1}\left(\operatorname{Hom}_{\rho_{0}, \theta}(\langle\theta\rangle, G)\right) .
$$

(A homomorphism belongs to $\operatorname{Hom}_{\Theta}(\Gamma, G)$ if and only if it sends each $\theta \in \Theta$ to $1 \in G$.) Therefore, $\operatorname{Hom}_{\Theta}(\Gamma, G)$ is also open in $\operatorname{Hom}(\Gamma, G)$.

2.2. Coxeter groups. We refer the reader to [3] for the basics of Coxeter groups.

Let $\Delta$ be a finite simplicial graph with the vertex and edge sets denoted $V=V(\Delta)$ and $E=E(\Delta)$ respectively. We will use the notation $e=[v, w]$ for the edge of $\Delta$ connecting $v$ and $w$, if it exists. We assume also that we are given a function

$$
m: E \rightarrow \mathbb{N}, \quad m(e) \geq 2, \forall e \in E,
$$


labeling the edges of $\Delta$. We will call the pair $(\Delta, m)$ a labeled graph or a Coxeter graph. Given a labeled graph, we define the associated Coxeter group $\Gamma=\Gamma_{\Delta}$ by the presentation

$$
\langle g_{v}, v \in V \mid \forall v, w \in V, g_{v}^{2}=1, \underbrace{g_{v} g_{w} \cdots}_{m \text { terms }}=\underbrace{g_{w} g_{v} \cdots}_{m \text { terms }}, e=[v, w], m=m(e), e \in E\rangle .
$$

Alternatively, one can describe the relators of this group as $g_{v}^{2}=1$ and

$$
\left(g_{v} g_{w}\right)^{m}=1
$$

where $m=m(e), e=[v, w]$.

Remark 2.5. Note that the notation we use here is different from the one in the Lie theory, where two generators commute whenever the corresponding vertices are not connected by an edge. In our notation, every such pair of elements of $\Gamma$ generates an infinite dihedral subgroup of $\Gamma$.

We also define the canonical central extension

$$
1 \rightarrow \mathbb{Z}_{2} \rightarrow \tilde{\Gamma} \stackrel{\eta}{\rightarrow} \Gamma \rightarrow 1
$$

of the group $\Gamma$, with the extended Coxeter group $\tilde{\Gamma}=\tilde{\Gamma}_{\Delta}$ given by the presentation

$$
\begin{gathered}
\left\langle z, g_{v}, v \in V\right| z^{2}=1, \forall v \in V,\left[g_{v}, z\right]=1, g_{v}^{2}=z, \\
\underbrace{g_{v} g_{w} \cdots}_{m \text { terms }}=z^{m+1} \underbrace{g_{w} g_{v} \cdots}_{m \text { terms }}, e=[v, w], m=m(e), e \in E\rangle .
\end{gathered}
$$

The number $r=|V|$ (the cardinality of $V$ ) is called the rank of $\Gamma$ and $\tilde{\Gamma}$. We will refer to the generator $z$ of the group $\Gamma$ as the central element of $\Gamma$, even though, the center of $\tilde{\Gamma}$ might be larger than $\mathbb{Z}_{2}$ : This happens precisely when $\Delta$ consists of a single vertex.

A subgraph $\Sigma \subset \Delta$ is called full if for every vertices $v, w \in \Sigma$, the edge $[v, w]$ in $\Delta$ also belongs to $\Sigma$. Every subgraph $\Sigma \subset \Delta$ inherits labels from $\Delta$. For the new labeled graph (which we still denote $\Sigma$ ), we have the natural homomorphism,

$$
\iota_{\Sigma}: \Gamma_{\Sigma} \rightarrow \Gamma_{\Delta}
$$

sending each generator $g_{v} \in \Gamma_{\Sigma}, v \in V(\Sigma)$, to the generator of $\Gamma_{\Delta}$ with the same name. It is immediate that the homomorphism $\iota_{\Sigma}$ lifts to a homomorphism

$$
\tilde{\iota}_{\Sigma}: \tilde{\Gamma}_{\Sigma} \rightarrow \tilde{\Gamma}_{\Delta}
$$

sending each $g_{v}$ to itself $(v \in V(\Sigma))$ and the central element $z \in \tilde{\Gamma}_{\Sigma}$ to the central element $z \in \tilde{\Gamma}_{\Delta}$. We will use this construction in two spacial cases:

a. $\Sigma:=\Delta_{\emptyset}$ is the subgraph which has the same vertex set as $\Gamma$, but empty edge set. Then

$$
\Gamma_{\Sigma} \cong F_{r}, \quad \tilde{\Gamma}_{\Sigma} \cong F_{r} \times \mathbb{Z}_{2}
$$

b. $\Sigma \subset \Delta$ is a full subgraph. In this case, the homomorphism $\iota_{\Sigma}$ is injective, see e.g. [3], page 113; it follows that the homomorphism $\tilde{\iota}_{\Sigma}$ is injective as well.

For full subgraphs $\Sigma \subset \Delta$, the subgroups $\iota_{\Sigma}\left(\Gamma_{\Sigma}\right)<\Gamma_{\Delta}$ and $\tilde{\iota}_{\Sigma}\left(\tilde{\Gamma}_{\Sigma}\right)<\tilde{\Gamma}_{\Delta}$ are called parabolic subgroups of $\Gamma_{\Delta}$ and $\tilde{\Gamma}_{\Delta}$ respectively. We say that a parabolic subgroup of $\Gamma_{\Delta}, \tilde{\Gamma}_{\Delta}$ is elementary, if it is a finite parabolic subgroup of rank $\leq 2$. The latter 
requirement simply means that $\Sigma$ consists of at most two vertices; the finiteness condition means that if $\Sigma$ consists of two vertices, then these vertices are connected by an edge. We will refer to such subgraphs $\Sigma$ elementary as well.

Example 2.6. 1. Suppose that $\Delta$ consists of a single edge $e$ labelled 2. Then $\Gamma_{\Delta} \cong \mathbb{Z}_{2} \times \mathbb{Z}_{2}$ and

$$
\tilde{\Gamma}_{\Delta} \cong Q_{8}
$$

the finite quaternion group.

2. If the edge $e$ is labeled 4 then $\Gamma_{\Delta}$ is the dihedral group $I_{2}(4)$ of order 8 ; it admits an epimorphism

$$
\Gamma_{\Delta} \rightarrow \mathbb{Z}_{2} \times \mathbb{Z}_{2}
$$

whose kernel is the center of $\Gamma_{\Delta}$, which is generated by the involution $g_{v} g_{w} g_{v} g_{w}$.

\section{Representations of Coxeter groups And extended Coxeter groups}

In this section we prove some basic facts about representations of Coxeter and extended Coxeter groups to $P S L(2, \mathbb{C})$ and $S L(2, \mathbb{C})$ respectively.

3.1. Representations of elementary Coxeter groups. Recall that the quotient map

$$
p: \tilde{G}(\mathbb{C})=S L(2, \mathbb{C}) \rightarrow G(\mathbb{C})=\operatorname{PSL}(2, \mathbb{C})=S L(2, \mathbb{C}) /\{ \pm 1\},
$$

is a 2 -fold covering. The extended Coxeter groups appear naturally in the context of lifting homomorphisms of Coxeter groups from $P S L(2, \mathbb{C})$ to $S L(2, \mathbb{C})$.

Consider the labelled graph $\Delta$, consisting of two vertices $v, w$ and the edge $[v, w]$ labelled $n \geq 2$. The corresponding Coxeter group $\Gamma_{\Delta}$ is a finite dihedral group, usually denoted $I_{2}(n)$. This group is isomorphic to the subgroup of the group of symmetries of a regular planar $2 n$-gon, acting simply transitively on the set of edges of this polygon. Hence, this group embeds canonically (up to conjugation) into $O(2)$ and, thus, into $P O(3, \mathbb{R}) \cong S O(3, \mathbb{R})<P S L(2, \mathbb{C})$. If $n$ is odd, then such group of symmetries will lift isomorphically to a subgroup of $S U(2)<S L(2, \mathbb{C})$. In contrast, we will be interested (only) in the case when $n$ is even; in fact, we will be using only the labels $n=2$ and $n=4$ in this paper.

Below we will prove several lemmas about faithful representations of elementary Coxeter (and extended Coxeter) groups into $G(\mathbb{C})$ (and $\tilde{G}(\mathbb{C})$ ).

Lemma 3.1. 1. There exists, unique up to conjugation, faithful representation $\rho: \mathbb{Z}_{2} \times \mathbb{Z}_{2} \rightarrow G(\mathbb{C})$.

2. There are no faithful representations $\mathbb{Z}_{2} \times \mathbb{Z}_{2} \rightarrow \tilde{G}(\mathbb{C})$.

Proof. 1. Since the image of $\rho$ is finite, its conjugate to a subgroup of $S O(3, \mathbb{R})<$ $G(\mathbb{C})$. Part 1 now follows from the fact that the group $S O(3, \mathbb{R})$ acts transitively on the set of pairs of orthogonal 1-dimensional subspaces of $\mathbb{R}^{3}$ (these subspaces, in our setting, are fixed lines of the images of the direct factors of $\mathbb{Z}_{2} \times \mathbb{Z}_{2}$ under $\rho$ ).

2. Part 2 follows from the fact that any involution $A \in S L(2, \mathbb{C})$ has both eigenvalues equal to -1 , i.e., $A$ equals $-1 \in S L(2, \mathbb{C})$.

The next lemma and proposition generalize Lemma 3.1 to representations of the dihedral group $I_{2}(4)$. 
Lemma 3.2. All injective representations $\rho: \Gamma=I_{2}(4) \rightarrow G(\mathbb{C})$ are conjugate to each other.

Proof. Since the group $\Gamma$ is finite, its image in $G(\mathbb{C})$ lies in a conjugate of the maximal compact subgroup $S O(3, \mathbb{R})<G(\mathbb{C})$. Thus, we will assume that $\rho(\Gamma)$ is contained in $S O(3, \mathbb{R})$. Since the product of the generating involutions $\rho\left(g_{v}\right), \rho\left(g_{w}\right)$ of $\rho(\Gamma)$ has order 4 , the fixed lines of $\rho\left(g_{v}\right), \rho\left(g_{w}\right)$ meet at the angle $\frac{\pi}{4}$ in $\mathbb{R}^{3}$. Now, the assertion follows from the fact that $S O(3, \mathbb{R})$ acts transitively on the set of 1dimensional subspaces in $\mathbb{R}^{3}$ meeting at the given angle.

Proposition 3.3. Consider the dihedral group $I_{2}(2 m)=\Gamma_{\Delta}$, and its isomorphism

$$
\rho: \Gamma_{\Delta} \rightarrow \Gamma<G(\mathbb{C}) .
$$

Then:

1. For every choice of matrices $\tilde{g}_{u} \in \tilde{G}(\mathbb{C})$ projecting to the generators $\rho\left(g_{u}\right) \in \Gamma<$ $G(\mathbb{C})$, the map

$g_{u} \rightarrow \tilde{g}_{u}, \quad u$
extends to a monomorphism $\tilde{\rho}: \tilde{\Gamma}_{\Delta} \rightarrow \tilde{G}(\mathbb{C})$.

2. The centralizer of the group $\tilde{\rho}\left(\tilde{\Gamma}_{\Delta}\right)$ in $\tilde{G}(\mathbb{C})$ equals the center of $\tilde{G}(\mathbb{C})$.

Proof. The proof amounts to elementary linear algebra, we include the details for the sake of completeness. For the notational convenience we will identify the isomorphic groups $\Gamma$ and $\Gamma_{\Delta}$. After conjugating the subgroup $\Gamma$ in $G(\mathbb{C})$, we can (and will) assume that $\Gamma$ lies in the subgroup $S O(3, \mathbb{R})<G(\mathbb{C})$. The orthogonal subgroup is covered by the unitary subgroup $S U(2)<S L(2, \mathbb{C})$. We let $Z(S U(2)) \cong \mathbb{Z}_{2}$ denote the center of $S U(2)$; this center consists of the matrices \pm 1 .

We begin with several trivial observations. Since $\rho$ is injective, the involutions $g_{v}, g_{w}$ are distinct rotations in $S O(3, \mathbb{R})$. In particular, their fixed-point sets in $\mathbb{C} P^{1}$ are pairwise disjoint. Suppose that the elements $\tilde{g}_{u}, \tilde{g}_{v} \in S U(2)$ project to $g_{v}, g_{w}$ respectively. Since the kernel of the covering $\tilde{G} \rightarrow G$ is isomorphic to $\mathbb{Z}_{2}$, the unitary transformations $\tilde{g}_{v}, \tilde{g}_{w} \in S U(2)$ have order at most 4 :

$$
\tilde{g}_{u}^{2} \in Z(S U(2)), u \in\{v, w\} .
$$

Note that the only involution in $S U(2)$ is the matrix -1 . Since $\tilde{g}_{u}$ projects nontrivially to $S O(3)$, this matrix cannot be an involution. It follows that

$$
\tilde{g}_{u}^{2}=-1 \in S U(2), \quad u \in\{v, w\} .
$$

The eigenvalues of the matrices $\tilde{g}_{v}, \tilde{g}_{w}$ have to be roots of unity of the order 4 , which implies that the spectrum of each matrix $\tilde{g}_{u}, u \in\{v, w\}$, equals $\{i,-i\}$.

We next claim that the eigenspaces of unitary transformations $\tilde{g}_{v}, \tilde{g}_{w}$ are pairwise distinct. If not, then these matrices would be simultaneously diagonalizable, which would imply that their projections to $\operatorname{PSL}(2, \mathbb{C})$ are equal. (Two involutions in $S O(3, \mathbb{R})$ which have same fixed point sets have to be the same.)

Suppose now that $A \in S L(2, \mathbb{C})$ is a matrix centralizing the subgroup $\left\langle\tilde{g}_{v}, \tilde{g}_{w}\right\rangle$ generated by $\tilde{g}_{v}, \tilde{g}_{w}$. We claim that $A$ is a scalar matrix, i.e., an element of the center of $S L(2, \mathbb{C})$. Indeed, since $A$ commutes with both $\tilde{g}_{v}, \tilde{g}_{w}$, it has to preserve the eigenspaces of each matrix $\tilde{g}_{v}, \tilde{g}_{w}$. (Here we are using the fact that the eigenvalues of $\tilde{g}_{u}$ are distinct, $u \in\{v, w\}$.) However, a nonscalar matrix in $S L(2, \mathbb{C})$ cannot have 
three distinct eigenlines. Therefore, $A$ is a scalar matrix. This implies the second claim of the lemma.

The generators $g_{v}, g_{w}$ satisfy

$$
t=\left(g_{v} g_{w}\right)^{m}=\left(g_{w} g_{v}\right)^{m}
$$

where $t$ is an order 2 element, which belongs to the center of $\Gamma$. (In the geometric realization of $\Gamma_{\Sigma}$ as a group of symmetries of a regular $2 n$-gon, the element $t$ corresponds to the order 2 rotation, the central symmetry of the polygon.)

If we had the relation

$$
\left(\tilde{g}_{v} \tilde{g}_{w}\right)^{m}=\left(\tilde{g}_{w} \tilde{g}_{w}\right)^{m}
$$

it would result in the monomorphism

$$
\alpha: \Gamma \rightarrow S U(2), \quad \alpha\left(g_{u}\right)=\tilde{g}_{u}, u \in\{v, w\},
$$

lifting the embedding $\rho: \Gamma \hookrightarrow S O(3, \mathbb{R})$. The image of the center $Z(\Gamma)$ of $\Gamma$ would then be in the center of $\alpha(\Gamma)$, hence, as we noted above, in the center of $S U(2)$. Then, the composition $\rho=p \circ \alpha$ would send $Z(\Gamma)$ to 1 , which is a contradiction.

This leaves us with the only possibility

$$
\left(\tilde{g}_{v} \tilde{g}_{w}\right)^{m}=-\left(\tilde{g}_{w} \tilde{g}_{v}\right)^{m} .
$$

To conclude, the map

$$
g_{v} \mapsto \tilde{g}_{v}, \quad z \mapsto-1 \in S L(2, \mathbb{C}),
$$

extends to an homomorphism $\tilde{\Gamma}_{\Delta} \rightarrow p^{-1}(\Gamma)$, sending the central element $z \in \tilde{\Gamma}_{\Delta}$ to the matrix $-1 \in S L(2, \mathbb{C})$. Injectivity of this homomorphism follows from injectivity of the representation $\Gamma \rightarrow P S L(2, \mathbb{C})$.

3.2. Representations faithful on elementary subgroups. For a Coxeter group $\Gamma=\Gamma_{\Delta}$ we define two subschemes:

$$
\operatorname{Hom}_{o}(\Gamma, G) \subset \operatorname{Hom}(\Gamma, G), \quad \operatorname{Hom}_{o}(\tilde{\Gamma}, \tilde{G}) \subset \operatorname{Hom}(\tilde{\Gamma}, \tilde{G}) .
$$

The former consists of homomorphisms which are injective on every elementary subgroup of $\Gamma$; the latter consists of homomorphisms which are injective on every elementary subgroup of $\tilde{\Gamma}$ and send $z \in \tilde{\Gamma}$ to $-1 \in S L(2, \mathbb{C}$ ). (In fact, the requirement for $z$ follows from faithfulness on elementary subgroups, except when $\Delta$ has no edges.) Since elementary subgroups of $\Gamma$ and $\tilde{\Gamma}$ are finite, both $\operatorname{Hom}_{o}(\Gamma, G)$ and $\operatorname{Hom}_{o}(\tilde{\Gamma}, \tilde{G})$ are open subschemes of the respective representation schemes. We will see later on that these subschemes are also closed. For each

$$
\rho \in \operatorname{Hom}_{o}(\tilde{\Gamma}, G)
$$

we have $\rho(z)=1$, while for each $\tilde{\rho} \in \operatorname{Hom}_{o}(\tilde{\Gamma}, \tilde{G})$ which projects to $\rho \in \operatorname{Hom}_{o}(\Gamma, G)$ we have $\tilde{\rho}(z)=-1$.

In the paper we will be using the labelled graph $\Omega$ depicted in the Figure 1: This graph has five vertices and nine edges. The edges left unlabeled in the figure, all have the label 2. The vertices $x, y$ are the only ones not connected to each other by an edge.

In what follows, we will also use the subgraph $\Upsilon \subset \Omega$, which is the complete graph on the vertices $u, v, w$. The parabolic subgroup $\Gamma_{\Upsilon}<\Gamma_{\Omega}$ is isomorphic to $\mathbb{Z}_{2}^{3}$. Since the group $\Gamma_{\Upsilon}$ is finite, the representation scheme $\operatorname{Hom}\left(\Gamma_{\Upsilon}, G\right)$ is smooth. 


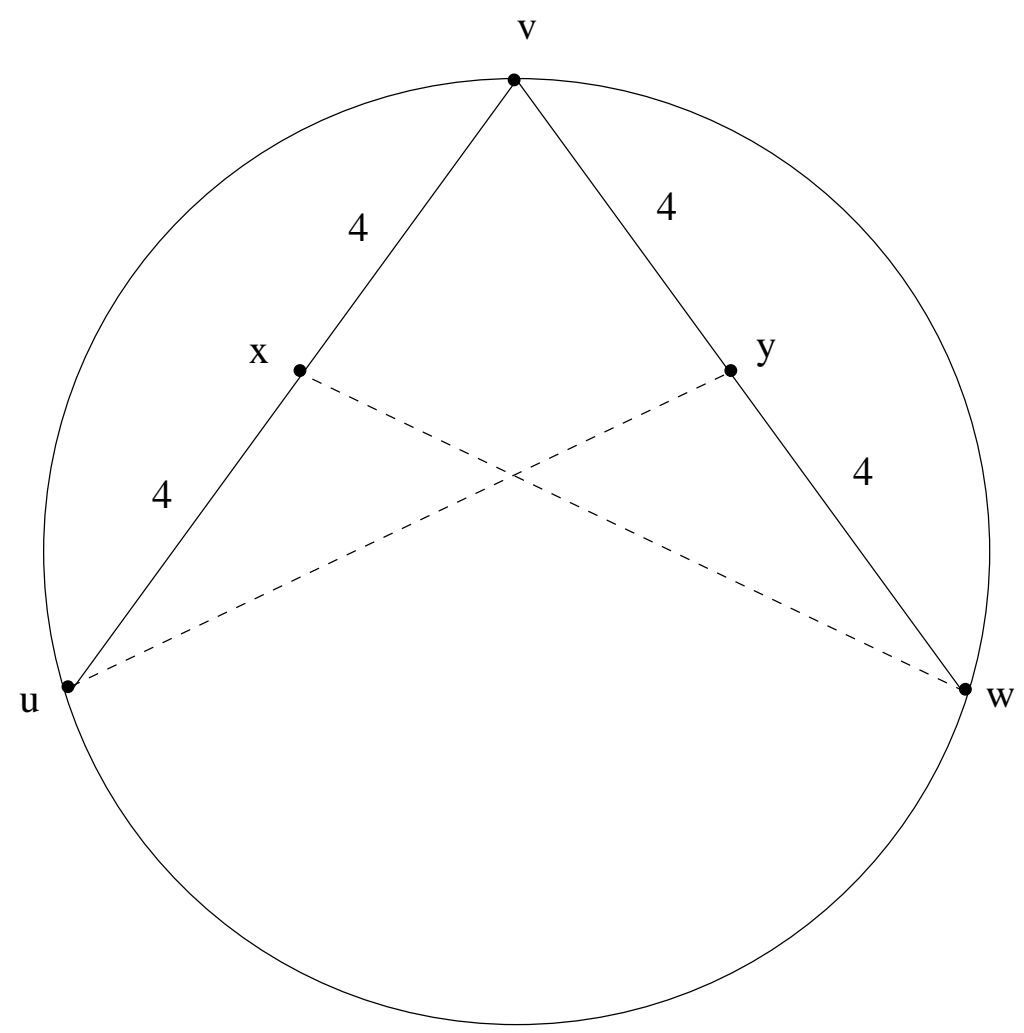

FiguRE 1. Graph $\Omega$.

Lemma 3.4. Each representation $\rho \in \operatorname{Hom}_{o}(\Gamma, G(\mathbb{C}))$ of the group $\Gamma=\Gamma_{\Upsilon}$ satisfies:

1. The kernel of $\rho$ is generated by the subgroup

$$
\left\langle g_{u} g_{v} g_{w}\right\rangle \cong \mathbb{Z}_{2}
$$

and the image of $\rho$ is isomorphic to $\mathbb{Z}_{2} \times \mathbb{Z}_{2}$.

2. The centralizer of the abelian subgroup $\rho(\Gamma)<G(\mathbb{C})$ in the group $G(\mathbb{C})$ equals the subgroup $\rho(\Gamma)$ itself.

3. $\operatorname{Hom}_{o}(\Gamma, G(\mathbb{C}))$ is the $G(\mathbb{C})$-orbit of a singleton $\left\{\rho_{\Upsilon}\right\}$.

Proof. This lemma is also elementary:

1. Consider a homomorphism $\rho \in \operatorname{Hom}_{o}(\Gamma, G(\mathbb{C}))$. For each element $\gamma \in \Gamma$ we let $\operatorname{Fix}(\gamma)$ denote the fixed-point set of $\rho(\gamma)$ in $\mathbb{C} P^{1}$. The condition that all three involutions $\rho\left(g_{u}\right), \rho\left(g_{v}\right), \rho\left(g_{w}\right)$ are distinct, implies that the three fixed-point sets Fix $\left(g_{u}\right)$, Fix $\left(g_{v}\right)$, Fix $\left(g_{w}\right)$ are pairwise disjoint. Commutativity of $\rho(\Gamma)$ implies that this group preserves the six-point set

$$
F=F i x\left(g_{u}\right) \cup F i x\left(g_{v}\right) \cup F i x\left(g_{w}\right) \subset \mathbb{C} P^{1} .
$$

The element $\rho\left(g_{u}\right)$ fixes Fix $\left(g_{u}\right)$, of course, and defines nontrivial involutions of the other two fixed-point sets

$$
F i x\left(g_{v}\right) \rightarrow F i g\left(g_{v}\right), \quad F i x\left(g_{w}\right) \rightarrow F i x\left(g_{w}\right) .
$$

The same applies to $g_{v}$ and $g_{w}$. It follows that

$$
\left.\rho\left(g_{u} g_{v}\right)\right|_{F}=\left.\rho\left(g_{w}\right)\right|_{F} .
$$


Hence,

$$
\rho\left(g_{u} g_{v}\right)=\rho\left(g_{w}\right)
$$

and, thus,

$$
\left\langle g_{u} g_{v} g_{w}\right\rangle<\operatorname{ker}(\rho)
$$

The equality of these subgroups of $\Gamma$ follows from the condition that

$$
\rho \in \operatorname{Hom}_{o}(\Gamma, G(\mathbb{C})) .
$$

This establishes Part 1 of the lemma.

2. To prove Part 2, note that every $g \in G$ centralizing $\rho(\Gamma)$ has to preserve each set Fix $\left(g_{u}\right)$, Fix $\left(g_{v}\right)$, Fix $\left(g_{w}\right)$. After composing $g$ with elements of $\rho(\Gamma)$, we achieve that $g$ fixes the set Fix $\left(g_{u}\right) \cup F i x\left(g_{v}\right)$ pointwise. Therefore, $g \in \rho(\Gamma)$. This proves 2 .

3. Part 3 note that, by Part 1, the pull-back morphism

$$
\operatorname{Hom}_{o}\left(\mathbb{Z}^{2}, G(\mathbb{C})\right) \rightarrow \operatorname{Hom}_{o}(\Gamma, G(\mathbb{C}))
$$

induced by the quotient homomorphism

$$
1 \rightarrow\left\langle g_{u} g_{v} g_{w}\right\rangle \rightarrow \Gamma \rightarrow \mathbb{Z}^{2}
$$

is surjective. Now, the claim follows from Lemma 3.1.

Lemma 3.5. 1. $\operatorname{Hom}_{o}\left(\Gamma_{\Omega}, G(\mathbb{C})\right)$ is a single orbit $G(\mathbb{C}) \cdot \rho_{\Omega}$.

2. The representation $\rho_{\Omega}$ is infinitesimally rigid: $\mathrm{H}^{1}\left(\Gamma_{\Omega}, \operatorname{sl}(2, \mathbb{C})\right)=0$.

3. For each $\rho \in \operatorname{Hom}_{o}\left(\Gamma_{\Omega}, G(\mathbb{C})\right)$, the adjoint action $A d \rho$ of $\Gamma_{\Omega}$ on the Lie algebra $\operatorname{sl}(2, \mathbb{C})$ has no nonzero fixed vectors.

Proof. 1. Consider $\rho \in \operatorname{Hom}_{o}\left(\Gamma_{\Omega}, G\right)$. In view of Lemma 3.4, we can assume that the restriction of $\rho$ to the subgroup $\Gamma_{\Upsilon}$ equals the representation $\rho_{\Upsilon}$. Consider now the dihedral subgroups

$$
\left\langle g_{u}, g_{x}\right\rangle, \quad\left\langle g_{x}, g_{v}\right\rangle
$$

in the group $\Gamma_{\Omega}$. It follows from Lemma 3.2 that there are exactly two extensions $\rho_{ \pm}$of the representation $\rho_{\Upsilon}$ to the subgroup $\left\langle g_{u}, g_{x}, g_{v}\right\rangle$ (which are faifthful on all elementary parabolic subgroups): For both representations, $\rho_{ \pm}\left(g_{x}\right)$ lies in $S O(3, \mathbb{R})$, its fixed line in $\mathbb{R}^{3}$ is contained in the span of the fixed lines of $\rho_{\Upsilon}\left(g_{u}\right), \rho_{\Upsilon}\left(g_{v}\right)$. This fixed line makes the angle $\frac{\pi}{4}$ with the fixed lines of $\rho_{\Upsilon}\left(g_{u}\right), \rho_{\Upsilon}\left(g_{v}\right)$ and is orthogonal to the fixed line of $\rho\left(g_{w}\right)$. These representations $\rho_{ \pm}$are conjugate via the element $\rho\left(g_{v}\right) \in S O(3)$. Therefore, after such conjugation, we fix the value $\rho\left(g_{x}\right)$. We next repeat this argument for the subgroup of $\Gamma_{\Omega}$ generated by

$$
\left\{g_{v}, g_{y}, g_{w}\right\} \text {. }
$$

Since conjugation via $\rho\left(g_{w}\right)$ does not alter $\rho\left(g_{x}\right)$, we obtain the required uniqueness statement.

2. In what follows we will be using the fact that the adjoint representation of $\operatorname{PSL}(2, \mathbb{C})$ is isomorphic to the complexification $V$ of the standard representation of $S O(3, \mathbb{R})$ on $\mathbb{R}^{3}$. We will also use the notation $V$ and $s l(2, \mathbb{C})$ for the representation $A d \rho$ of the group $\Gamma_{\Omega}$ (and its subgroups) in the notation for cocycles and cohomology groups. In particular, for each element $a$ of

$$
\{u, v, w, x, y\}
$$


the fixed-point set of $A d \rho\left(g_{a}\right)$ is a line in $V$, which we will denote by $V^{a}$. An elementary but useful geometric observation is that

$$
V^{x} \subset V^{u} \oplus V^{v}
$$

while

$$
V=V^{u} \oplus V^{v} \oplus V^{w}=V^{u} \oplus V^{x} \oplus V^{w} .
$$

Consider a cocycle $\xi \in Z^{1}\left(\Gamma_{\Omega}, V\right)$. Since $\Gamma_{\Upsilon}$ is finite, $\mathrm{H}^{1}\left(\Gamma_{\Upsilon}, V\right)=0$. Since the restriction of $\xi$ to the subgroup $\Gamma_{\Upsilon}$ is a coboundary, by subtracting off a coboundary from $\xi$, we can assume that $\xi$ vanishes in $\Gamma_{\Upsilon}$. Similarly, there exist $\alpha, \beta \in V$ such that

$$
\begin{aligned}
& \xi(h)=\alpha-A d \rho(h) \alpha, \quad \forall h \in\langle x, u\rangle \\
& \xi(h)=\beta-A d \rho(h) \beta, \quad \forall h \in\langle x, w\rangle .
\end{aligned}
$$

It follows that $\alpha \in V^{u}, \beta \in V^{w}$. Moreover, by looking at the value $\xi(x)$, we see that

$$
\alpha-\beta \in V^{x} \text {. }
$$

Since the lines $V^{u}, V^{x}, V^{w}$ also span $V$, it follows that $\alpha=\beta=0$. Therefore, $\xi(x)=0$. Similarly, $\xi(y)=0$ and, thus, $\xi=0$ on the entire group $\Gamma_{\Omega}$.

3. This follows from the fact that $\rho\left(\Gamma_{\Upsilon}\right)$ has no nonzero fixed vectors in $V=$ $\operatorname{sl}(2, \mathbb{C})$.

Corollary 3.6. The scheme Hom ${ }_{o}\left(\Gamma_{\Omega}, G\right)$ is smooth.

From now on, we will be making the following assumption on the labeled graphs $\Delta$ of Coxeter groups $\Gamma$ :

Assumption 3.7. 1. Every label of the graph $\Delta$ is even.

2. $\Delta$ contains as a full subgraph the graph $\Omega$ above.

Proposition 3.8. 1. The schemes $\operatorname{Hom}_{o}(\Gamma, G)$ and $\operatorname{Hom}_{o}(\tilde{\Gamma}, \tilde{G})$ are clopen 1 subschemes in $\operatorname{Hom}(\Gamma, G)$ and $\operatorname{Hom}(\tilde{\Gamma}, \tilde{G})$ respectively.

2. There is a morphism of schemes $q: \operatorname{Hom}_{o}(\tilde{\Gamma}, \tilde{G}) \rightarrow \operatorname{Hom}_{o}(\Gamma, G)$, such that for every $\tilde{\rho} \in \operatorname{Hom}_{o}(\tilde{\Gamma}, \tilde{G})$ and $\rho=q(\tilde{\rho})$ we have

$$
p \circ \tilde{\rho}=\rho \circ \eta,
$$

where $\eta: \tilde{\Gamma} \rightarrow \Gamma$ is the quotient map from (1).

3. The morphism $q$ is a regular étale covering with the deck-group $\mathbb{Z}_{2}^{r}$, where $r$ is the rank of $\Gamma$.

Proof. 1. We will give a proof for $\operatorname{Hom}_{o}(\Gamma, G)$, since the other statement is similar. Consider an elementary subgroup $\Gamma_{\Sigma} \subset \Gamma$; this subgroup is finite. In Lemma 2.1 we proved that each irreducible component of $\operatorname{Hom}\left(\Gamma_{\Sigma}, G\right)$ is a clopen subscheme of $\operatorname{Hom}\left(\Gamma_{\Sigma}, G\right)$; furthermore, each component is a single $G$-orbit of a representation $\Gamma_{\Sigma} \rightarrow G$. Then

$$
\operatorname{Hom}_{o}\left(\Gamma_{\Sigma}, G\right)=\operatorname{Hom}\left(\Gamma_{\Sigma}, G\right) \backslash \bigcup_{\theta \in \Gamma_{\Sigma}-\{1\}} \operatorname{Hom}_{\langle\theta\rangle}\left(\Gamma_{\Sigma}, G\right)
$$

\footnotetext{
${ }^{1}$ I.e. closed and open.
} 
is an open subscheme in $\operatorname{Hom}\left(\Gamma_{\Sigma}, G\right)$. It is also closed since every subscheme removed was open.

For each elementary subgroup $\Gamma_{\Sigma}<\Gamma$ and the inclusion map $\iota_{\Sigma}: \Gamma_{\Sigma} \rightarrow \Gamma$, we have the pull-back morphism

$$
\iota_{\Sigma}^{*}: \operatorname{Hom}(\Gamma, G) \rightarrow \operatorname{Hom}\left(\Gamma_{\Sigma}, G\right) .
$$

Then we have the finite intersection, taken over all elementary subgraphs $\Sigma \subset \Delta$ :

$$
\operatorname{Hom}_{o}(\Gamma, G)=\bigcap_{\Sigma}\left(\phi_{\Sigma}^{*}\right)^{-1}\left(\operatorname{Hom}_{o}\left(\Gamma_{\Sigma}, G\right)\right) .
$$

Therefore, $\operatorname{Hom}_{o}(\Gamma, G) \subset \operatorname{Hom}(\Gamma, G)$ is clopen as a finite intersection of clopen subschemes.

2. For each $\tilde{\rho} \in \operatorname{Hom}_{o}(\tilde{\Gamma}, \tilde{G}(\mathbb{C}))$, the reduction modulo centers of $\tilde{\Gamma}$ and $\tilde{G}$ yields a homomorphism $\rho \in \operatorname{Hom}_{o}(\Gamma, G(\mathbb{C}))$. We need to check that the map

$$
q: \operatorname{Hom}_{o}(\tilde{\Gamma}, \tilde{G}(\mathbb{C})) \rightarrow \operatorname{Hom}_{o}(\Gamma, G(\mathbb{C})), \quad q(\tilde{\rho})=\rho
$$

obtained in this fashion comes from a morphism of schemes. First, the composition

$$
\tilde{\rho} \rightarrow p \circ \rho, \quad \operatorname{Hom}(\tilde{\Gamma}, \tilde{G}) \rightarrow \operatorname{Hom}(\tilde{\Gamma}, G)
$$

is clearly a morphism of schemes. For $\Theta=\{z\}$, we obtain an isomorphism of the schemes

$$
\operatorname{Hom}_{\Theta}(\tilde{\Gamma}, G) \rightarrow \operatorname{Hom}(\Gamma, G),
$$

see Lemma 2.3. and $\operatorname{Hom}_{\Theta}(\tilde{\Gamma}, G)$ contains the image of $\operatorname{Hom}_{o}(\tilde{\Gamma}, \tilde{G})$. Therefore, $q$ is a composition of two morphisms.

We next verify surjectivity. Let $\rho \in \operatorname{Hom}_{o}(\Gamma, G)$. Define $\tilde{\rho}: \tilde{\Gamma} \rightarrow \tilde{G}$ by sending generators $g_{v}$ to arbitrary elements of $p^{-1}\left(\rho\left(g_{v}\right)\right)$ and sending the central element $z \in \tilde{\Gamma}$ to $-1 \in S L(2, \mathbb{C})$. In view of Proposition 3.3. for each elementary subgroup $\Gamma_{\Sigma}$ in $\Gamma$, the restriction of $\tilde{\rho}$ to the generators of $\tilde{\Gamma}_{\Sigma}$ extends to a faithful homomorphism $\left.\tilde{\rho}\right|_{\Gamma_{\Sigma}}$.

Since all the relators of the group $\tilde{\Gamma}$ come from elementary subgroups, it follows that our map of the generators of $\tilde{\Gamma}$ to $S L(2)$ extends to a homomorphism $\tilde{\rho}: \tilde{\Gamma} \rightarrow S L(2)$. This homomorphism belongs to $\operatorname{Hom}_{o}(\tilde{\Gamma}, \tilde{G})$ since it is faithful on each elementary subgroup.

Thus, we obtained a surjective morphism

$$
q: \operatorname{Hom}_{o}(\tilde{\Gamma}, \tilde{G}) \rightarrow \operatorname{Hom}_{o}(\Gamma, G), q(\tilde{\rho})=\rho .
$$

The group $\mathbb{Z}_{2}$ is the group of automorphisms of the covering $\tilde{G} \rightarrow G$; therefore, the product of $r$ copies of $\mathbb{Z}_{2}$ acts naturally on the product of $r$ copies of $\tilde{G}$ as the group of automorphisms of the (regular) cover

$$
\hat{p}=p \times \ldots \times p: \prod_{i=1}^{r} \tilde{G} \rightarrow \prod_{i=1}^{r} G .
$$

Since the rank $r$ of the group $\Gamma$ is the number of its generators $g_{v}$, we have the morphism

$$
\prod_{i=1}^{r} \tilde{G} \cong \operatorname{Hom}\left(F_{r}, \tilde{G}\right) \stackrel{\hat{p}}{\longrightarrow} \prod_{i=1}^{r} G \cong \operatorname{Hom}\left(F_{r}, G\right)
$$


where $F_{r}$ is the free group on $r$ generators. We also have the following commutative diagram

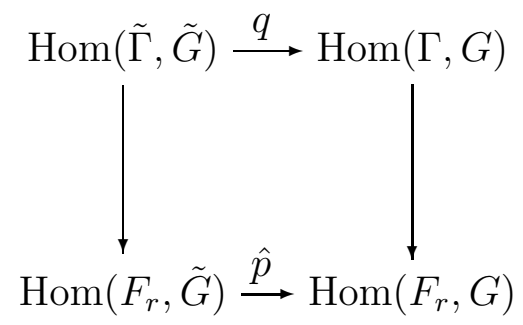

where the vertical arrows are the inclusions of representation schemes induced by the epimorphisms

$$
F_{r} \rightarrow \tilde{\Gamma}, \quad F_{r} \rightarrow \Gamma
$$

sending the free generators of $F_{r}$ to the generators $g_{r}$ of extended Coxeter and Coxeter groups. It is elementary and left to the reader to verify that the group $\mathbb{Z}_{2}^{r}$ of automorphisms of $\hat{p}$ preserves the subscheme $\operatorname{Hom}_{o}(\tilde{\Gamma}, \tilde{G})$. Therefore, this finite group acts simply transitively on the fibers of the morphism $q$.

3. It remains to show that the map $q$ is etale, i.e., is an isomorphism of analytic germs at every point. Let $\tilde{\rho}$ be in $\operatorname{Hom}_{o}(\tilde{\Gamma}, S L(2, \mathbb{C}))$ and set $\rho:=q(\tilde{\rho})$.

Below is a proof which assumes reader's familiarity with [1], where the theory of controlling differential graded Lie algebras for various deformation problems was developed.

In view of [1, Theorem 6.8], it suffices to verify that the differential graded Lie algebras controlling these germs are quasi-isomorphic. First, the Lie algebras of $G$ and $\tilde{G}$ are isomorphic under the covering $p$, which implies that the covering map $p$ induces an isomorphism

$$
\mathrm{H}^{i}(\tilde{\Gamma}, A d \circ \tilde{\rho}) \rightarrow \mathrm{H}^{i}(\tilde{\Gamma}, A d \circ p(\tilde{\rho})), i \geq 0 .
$$

Since the central subgroup $\mathbb{Z}_{2}$ of $\tilde{\Gamma}$ is finite,

$$
\mathrm{H}^{i}\left(\mathbb{Z}_{2}, \operatorname{sl}(2, \mathbb{C})\right)=0, \quad i \geq 1 \text {. }
$$

Therefore, applying the Lyndon-Hochshild-Serre spectral sequence to the central extension (1), we obtain isomorphisms

$$
\mathrm{H}^{i}(\Gamma, A d \circ \rho) \rightarrow \mathrm{H}^{i}(\tilde{\Gamma}, A d \circ \tilde{\rho}), \rho=q(\tilde{\rho}), i \geq 1 .
$$

(Actually, for $i=0$ both cohomology groups vanish, which implies that they are also isomorphic.) These isomorphisms ensure that the morphism

$$
q:(\operatorname{Hom}(\tilde{\Gamma}, \tilde{G}), \tilde{\rho}) \rightarrow(\operatorname{Hom}(\Gamma, G), \rho)
$$

is an isomorphisms of germs.

Remark 3.9. Below is an alternative argument proving that $q$ is etale, which does not reply upon differential graded Lie algebras. The morphism $q$ is etale if and only if $q$ induces bijections of sets of $A$-points of representation schemes for all local Artin $\mathbb{C}$-algebras $A$. Let $A$ be a local Artin $\mathbb{C}$-algebra and $\epsilon: A \rightarrow \mathbb{C}$ be the quotient by 
the maximal ideal. Then we have natural bijections $\operatorname{Hom}(\Gamma, G(A)) \cong \operatorname{Hom}(\Gamma, G)(A)$ and $\operatorname{Hom}(\tilde{\Gamma}, \tilde{G}(A)) \cong \operatorname{Hom}(\tilde{\Gamma}, \tilde{G})(A)$, and the commutative diagram

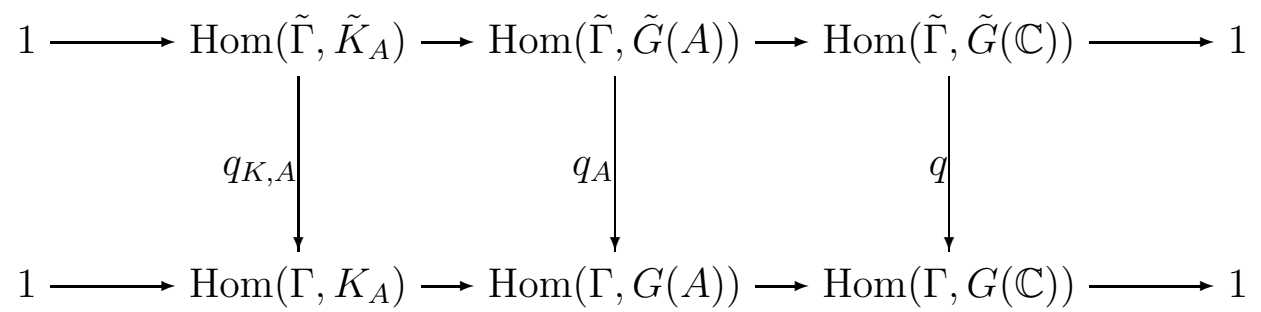

where $\tilde{K}_{A}$ and $K_{A}$ are the respective kernels of the group homomorphisms

$$
\tilde{G}(A) \rightarrow \tilde{G}(\mathbb{C}), \quad G(A) \rightarrow G(\mathbb{C})
$$

induced by $\epsilon: A \rightarrow \mathbb{C}$. We observe that the group $\tilde{K}_{A}$ is torsion free nilpotent and, since the covering map $p: \tilde{G} \rightarrow G$ is etale, the induced map $p_{K, A}: \tilde{K}_{A} \rightarrow K_{A}$ is an isomorphism. Since $\tilde{K}_{A}$ is torsion free, each homomorphism $\tilde{\rho}_{A}: \tilde{\Gamma} \rightarrow \tilde{G}(A)$ descends to a canonical homomorphism $\rho_{A}: \Gamma \rightarrow G(A)$. The fact that $\tilde{K}_{A}$ is torsion-free also implies that if $\tilde{\rho}_{A}, \tilde{\rho}_{A}^{\prime}$ have the same projection to $\operatorname{Hom}(\tilde{\Gamma}, \tilde{G}(\mathbb{C}))$ and define the same homomorphism $\rho_{A}: \Gamma \rightarrow G(A)$, then $\tilde{\rho}_{A}=\tilde{\rho}_{A}^{\prime}$. Therefore, $q_{A}$ is a injective. The proof that $q_{A}$ is surjective is also elementary and is left to the reader.

3.3. Character schemes of representations faithful on elementary subgroups. In this section we extend the results of the previous section from representation schemes to character schemes.

3.3.1. Stability. Given a reductive affine algebraic group $H$ and a finitely-generated group $\Lambda$, we have the algebraic action of the group $H$ on the homomorphism scheme $\operatorname{Hom}(\Lambda, H)$,

$$
(h, \rho) \mapsto \operatorname{Inn}(h) \circ \rho,
$$

where $\operatorname{Inn}(h)$ is the inner automorphism $g \mapsto h g h^{-1}$ of the group $H$. Recall that the character scheme is defined as the Mumford quotient

$$
X(\Lambda, H)=\operatorname{Hom}(\Lambda, H) / / H \text {. }
$$

Geometrically speaking, Mumford quotient is obtained by identifying semistable points $\operatorname{Hom}_{s s}(\Lambda, H)$ of the $H$-action by the extended orbit equivalence relation, while the restriction of the projection

$$
\mu: \operatorname{Hom}_{s s}(\Lambda, H) \rightarrow X(\Lambda, H)
$$

to the stable locus $\operatorname{Hom}_{s t}(\Lambda, H)$ (consisting of stable points) is just the quotient by the $H$-orbit equivalence. Hence the restriction of the projection to the stable locus has especially simple form. We will use the notation

$$
\rho \mapsto[\rho]
$$

for the projection $\mu$.

A sufficient condition for stability of representations $\rho \in \operatorname{Hom}(\Lambda, H)$ (under the $H$-action) in terms of the Zariski closure of $\rho(\Lambda)$ in $H$ was established in [10]:

Theorem 3.10. A representation $\rho \in \operatorname{Hom}(\Lambda, H)$ is semistable provided that the Zariski closure $\overline{\rho(\Lambda)}$ is reductive. A representation is stable provided that the Zariski closure $\overline{\rho(\Lambda)}$ is reductive and the centralizer $Z_{H}(\rho(\Lambda))$ of the image of $\rho$ is finite. 
In the case of representations into $H=P O(3)$ and $H=\operatorname{Spin}(3)$, the sufficient condition for stability amounts to requiring that the image of $\rho$ is not contained in a Borel subgroup of $H$. Our next goal is to verify stability condition and identify centralizers of the images of representations in the context of Coxeter and extended Coxeter groups. Recall that we are using the notation $G$ for $P S L(2)$ and $\tilde{G}$ for $S L(2)$ (regarded as group-schemes).

Lemma 3.11. Let $\Gamma$ be a Coxeter group and $\tilde{\Gamma}$ the corresponding extended Coxeter group, satisfying the Assumption 3.7. Then for each $\rho \in \operatorname{Hom}_{o}(\Gamma, G)$ and $\tilde{\rho} \in \operatorname{Hom}_{o}(\tilde{\Gamma}, \tilde{G})$ we have:

1. The representations $\rho, \tilde{\rho}$ are stable points in Mumford's sense.

2. The centralizers of the images of $\rho, \tilde{\rho}$ equal the center of the target group.

Proof. Recall that we require the group $\Gamma$ to contain a subgroup $\Gamma_{\Omega}$. It suffices to prove both 1 and 2 for the representations $\rho \in \operatorname{Hom}_{o}\left(\Gamma_{\Omega}, G\right), \tilde{\rho} \in \operatorname{Hom}_{o}\left(\tilde{\Gamma}_{\Omega}, \tilde{G}\right)$, since we have to verify that the image of the representation is not contained in a Borel subgroup and that its centralizer equals the center of the target group.

(i) First, we consider the case of representations $\tilde{\rho}: \tilde{\Gamma}=\tilde{\Gamma}_{\Omega} \rightarrow S L(2, \mathbb{C})$. We restrict our attention to the subgraph $\Sigma \subset \Omega$, which consists of two vertices $x, y$ and the edge $e=[x, y]$ labelled 4. Each representation $\tilde{\rho} \in \operatorname{Hom}_{o}(\tilde{\Gamma}, \tilde{G})$ projects to a faithful representation

$$
\rho: \Gamma_{\Sigma} \hookrightarrow P S L(2, \mathbb{C}) .
$$

By Proposition 3.3, the centralizer of the subgroup $\tilde{\rho}\left(\tilde{\Gamma}_{\Sigma}\right)$ equals the center of $S L(2, \mathbb{C})$. Moreover, the images of the generators of $\tilde{\Gamma}_{\Sigma}$ under $\tilde{\rho}$ have distinct eigenlines. It follows that the subgroup $\tilde{\rho}\left(\tilde{\gamma}_{\Sigma}\right)$ cannot have an invariant line in $\mathbb{C}^{2}$, thereby proving that $\tilde{\rho}(\tilde{\Gamma})$ is not contained in a Borel subgroup of $S L(2, \mathbb{C})$. This proves Parts 1 and 2 for representations to $S L(2, \mathbb{C})$.

(ii) Consider now representations $\rho: \Gamma \rightarrow G$. By the assumption, $\rho$ sends distinct generators of $\Gamma$ to distinct elements of $G$. It follows that the group $\rho(\Gamma)$ cannot fix a point in $\mathbb{C} P^{1}$. In other words, the group $\rho(\Gamma)$ is not contained in a Borel subgroup of $G$. This proves Part 1 .

To prove Part 2, we will use subgroups $\Gamma_{\Upsilon}$ and $\Gamma_{\Omega}$ of the group $\Gamma$. Since $\rho$ belong to $\operatorname{Hom}_{o}(\Gamma, P S L(2, \mathbb{C}))$, the centralizer of $\rho\left(\Gamma_{\Upsilon}\right)$ in $G$ equals the subgroup $\rho\left(\Gamma_{\Upsilon}\right)$ itself (Lemma 3.4). On the other hand, $\rho$ is faithful on the subgroups generated by $\left\{g_{u}, g_{x}\right\},\left\{g_{v}, g_{y}\right\},\left\{g_{w}, g_{z}\right\}$. Therefore,

$$
\left[\rho\left(g_{u}\right), \rho\left(g_{x}\right)\right] \neq 1, \quad\left[\rho\left(g_{v}\right), \rho\left(g_{y}\right)\right] \neq 1, \quad\left[\rho\left(g_{w}\right), \rho\left(g_{z}\right)\right] \neq 1,
$$

and, hence, the subgroup $\rho(\Gamma)$ has trivial centralizer in $P S L(2, \mathbb{C})$.

3.3.2. Cross-sections. Let $Y$ be an (quasiaffine) scheme and $G \curvearrowright Y$ be a stable (in Mumford's sense) algebraic group action on $Y$. Suppose that $S \subset Y$ is a closed subscheme, such that the orbit map

$$
G \times S \rightarrow Y
$$

is an isomorphism. (In particular, $S$ projects isomorphically onto $Y / / G$ ). Then $S$ is called a cross-section for the action of $G$ on $Y$. 
Lemma 3.12. Suppose that $Y$ is a (quasiaffine) scheme of finite type, $G \times Y \rightarrow Y$ is an (algebraic) action of an affine algebraic group, $S \subset X$ is a cross-section for this action. Suppose that $Y^{\prime} \subset Y$ is a $G$-invariant subscheme. Then $S^{\prime}=Y^{\prime} \cap S$ is also a cross-section for the action $G \times Y^{\prime} \rightarrow Y^{\prime}$.

Proof. We need to show that the orbit map $G \times S^{\prime} \rightarrow Y^{\prime}$ is an isomorphism. It suffices to show that for each commutative ring $A$, the orbit map

$$
\mu^{\prime}: G(A) \times S^{\prime}(A) \rightarrow Y^{\prime}(A)
$$

of $A$-points is a bijection. We have $S^{\prime}(A)=S(A) \cap Y(A)$. Since the orbit map

$$
\mu: G(A) \times S(A) \rightarrow Y(A)
$$

is a bijection and $Y^{\prime}(A)$ is $G(A)$-invariant, it follows that $\mu$ is a bijection.

Note that if the scheme $Y$ and its subscheme $S \subset Y$ are both smooth then the condition that $S$ is a cross-section for the action of $G$ is easier to check: It suffices to verify that the set of complex points of $S$ is a set-theoretic cross-section for the action of $G$ and that $G$-orbits are transversal to $S$ : For each $y \in S(\mathbb{C})$,

$$
T_{y} Y \cong T_{y}(G y) \oplus T_{y} S
$$

We now specialize to the case of representation schemes. Let $\pi^{\prime}=\pi / N$ be a finitely-generated group (where $\pi$ is a finitely generated group and $N \triangleleft \pi$ is a normal subgroup), $G$ is an affine algebraic group $G \times \operatorname{Hom}(\pi, G) \rightarrow \operatorname{Hom}(\pi, G)$ is the action of $G$ by conjugation on the representation scheme. We will think of $\operatorname{Hom}(\pi, G)$ as a closed subscheme in the smooth scheme $\operatorname{Hom}(F, G)$. Suppose that $U \subset \operatorname{Hom}(F, G)$ is a $G$-invariant open affine subscheme and $U^{\prime}=U \cap \operatorname{Hom}(\pi, G)$. We assume that $S \subset U$ is a closed smooth subscheme. Then, in view of smoothness, the property that $S$ is a cross-section for the $G$-action amounts to:

1. $S(\mathbb{C})$ is a cross-section for the action of $G(\mathbb{C})$ on $U$.

2. For each $\rho \in S(\mathbb{C})$ the action of $\rho(\pi)$ on the Lie algebra of $G(\mathbb{C})$ has no nonzero invariant vectors. (This condition amounts to the transversality property above.)

In view of Lemma 3.12, the subscheme $S^{\prime}=S \cap U^{\prime}$ is a cross-section for the action of $G$ on $U^{\prime}$.

3.3.3. Cross-sections of representation schemes. We apply the above observations in two situations. First, suppose that $\Gamma$ is a Coxeter group satisfying the Assumption 3.7, we let $G=P O(3)$. We have the identity embedding $\iota_{\Omega}: \Gamma_{\Omega} \hookrightarrow \Gamma$ of the finite subgroup $\Gamma_{\Omega}$. Recall that, according to Lemma 3.5. $\operatorname{Hom}_{o}\left(\Gamma_{\Omega}, G(\mathbb{C})\right)$ consists of a single $G$-orbit $G(\mathbb{C}) \cdot \rho_{\Omega}$. We then set

$$
\operatorname{Hom}_{c}(\Gamma, G):=\left(\iota_{\Omega}^{*}\right)^{-1}\left(\rho_{\Omega}\right) .
$$

The next lemma is an analogue of Corollary 12.11 in [5]:

Lemma 3.13. The subscheme $\operatorname{Hom}_{c}(\Gamma, G)$ is a cross-section for the action $G \curvearrowright$ $\operatorname{Hom}_{o}(\Gamma, G)$. In particular,

$$
X_{o}(\Gamma, G) \cong \operatorname{Hom}_{c}(\Gamma, G)
$$


Proof. We let $\pi^{\prime}=\Gamma$ and define the new group $\pi$ as the Coxeter group whose Coxeter graph is obtained from the one of $\Gamma$ by removing all the edges which are not in $\Omega$. Define

$$
\pi_{o}:=\mathbb{Z}_{2} \star \ldots \star \mathbb{Z}_{2} .
$$

Then the representation scheme $\operatorname{Hom}_{o}(\pi, G)$ is smooth, since $\pi$ is isomorphic to the free product

$$
\pi_{o} \star \Gamma_{\Omega},
$$

and $U=\operatorname{Hom}_{o}\left(\Gamma_{\Omega}, G\right)$ is smooth by Lemma 3.5. Clearly, $\pi^{\prime}=\pi / N$ for a normal subgroup $N \triangleleft \pi$. We again have the inclusion homomorphism $\iota_{\Upsilon}: \Gamma_{\Omega} \rightarrow \pi$; the subscheme

$$
S=\operatorname{Hom}_{c}(\pi, G):=\left(\iota_{\Upsilon}^{*}\right)^{-1}\left(\rho_{\Omega}\right)
$$

is smooth since it is naturally isomorphic to $\operatorname{Hom}\left(\pi_{o}\right)$. The fact that $S$ is a crosssection for the action of $G$ on $U$ follows immediately from Lemma 3.5 and observations following Lemma 3.12. Lastly, note that

$$
U^{\prime}=\operatorname{Hom}_{c}(\Gamma, G)=U \cap \operatorname{Hom}(\Gamma, G) .
$$

Now, the lemma follows from Lemma 3.12

The second situation when we apply our description of cross-sections is the one of representations of extended Coxeter groups $\tilde{\Gamma}$ (again satisfying the Assumption 3.7) to the group $\tilde{G} \cong S L(2)$. The group $\tilde{G}$ does not act faithfully on $\operatorname{Hom}(\tilde{\Gamma}, \tilde{G})$; this action factors through the action of the group $G=P O(3)$.

Earlier, we defined the subscheme $\operatorname{Hom}_{o}(\tilde{\Gamma}, \tilde{G}) \subset \operatorname{Hom}(\tilde{\Gamma}, \tilde{G})$. Set

$$
\operatorname{Hom}_{c}(\tilde{\Gamma}, \tilde{G}):=q^{-1}\left(\operatorname{Hom}_{c}(\Gamma, G)\right) .
$$

Lemma 3.14. $\operatorname{Hom}_{c}(\tilde{\Gamma}, \tilde{G})$ is a cross-section for the action of $G$ on $\operatorname{Hom}_{o}(\tilde{\Gamma}, \tilde{G})$.

Proof. We let $\pi^{\prime}=\tilde{\Gamma}$. Similarly to the proof of Lemma 3.13, we define the extended Coxeter group $\pi$ by eliminating all the edges which are not in the subgraph $\Omega$. Then $\pi^{\prime}$ is isomorphic to a quotient of $\pi$ and the same proof as in Lemma 3.13 goes through.

3.3.4. Character schemes. We let $X_{o}(\Gamma, G)$ and $X_{o}(\tilde{\Gamma}, \tilde{G})$ denote the projections of $\operatorname{Hom}_{o}(\Gamma, G)$ and $\operatorname{Hom}_{o}(\tilde{\Gamma}, \tilde{G})$ to the corresponding character schemes.

In view of Lemmata Lemma 3.13 and Lemma 3.14, the projections

$$
\operatorname{Hom}_{o}(\Gamma, G) \rightarrow X_{o}(\Gamma, G), \quad \operatorname{Hom}_{o}(\tilde{\Gamma}, \tilde{G}) \rightarrow X_{o}(\tilde{\Gamma}, \tilde{G})
$$

are principal fiber bundles with the structure group $G=P S L(2, \mathbb{C})$ : The center of the group $\tilde{G}$ acts trivially on $\operatorname{Hom}(\tilde{\Gamma}, \tilde{G})$. We record this as

Corollary 3.15. There exist natural isomorphisms of germs

$$
\begin{gathered}
\left(\operatorname{Hom}_{o}(\tilde{\Gamma}, S L(2)), \tilde{\rho}\right) \cong\left(\operatorname{Hom}_{c}(\tilde{\Gamma}, S L(2)) \times P S L(2), \tilde{\rho} \times 1\right) \cong \\
\left(X_{o}(\tilde{\Gamma}, S L(2)) \times P S L(2),[\tilde{\rho}] \times 1\right)
\end{gathered}
$$

and

$$
\begin{gathered}
\left.\left(\operatorname{Hom}_{o}(\Gamma, P S L(2)), \rho\right) \cong \operatorname{Hom}_{c}(\Gamma, P S L(2)) \times P S L(2), \rho \times 1\right) \cong \\
\left(X_{o}(\Gamma, P S L(2)) \times P S L(2),[\rho] \times 1\right) .
\end{gathered}
$$


3.3.5. Adding a free factor. Let $F_{k}$ be the free group on $k$ generators. For an arbitrary finitely-generated group $\Lambda$ and an algebraic group $H$ we have an isomorphism of schemes:

$$
\operatorname{Hom}\left(\Lambda \star F_{k}, H\right) \cong \operatorname{Hom}(\Lambda, H) \times \operatorname{Hom}\left(F_{k}, H\right) \cong \operatorname{Hom}(\Lambda, H) \times H^{k} .
$$

This isomorphism is $H$-equivariant, where the action of $H$ by conjugation on the left side and the diagonal action (by conjugations) on the product space on the right side. We will use these isomorphisms in the two spacial cases: $\Lambda=\Gamma, H=G$ and $\Lambda=\tilde{\Gamma}, H=\tilde{G}$, where $G=\operatorname{PSL}(2, \mathbb{C}), \tilde{G}=S L(2, \mathbb{C})$ and $\Gamma, \tilde{\Gamma}$ are Coxeter and extended Coxeter groups respectively. Then the isomorphisms (3) for these groups allow us to define clopen subschemes

$$
\operatorname{Hom}_{o}\left(\Gamma \star F_{k}, G\right) \subset \operatorname{Hom}\left(\Gamma \star F_{k}, G\right), \quad \operatorname{Hom}_{o}\left(\tilde{\Gamma} \star F_{k}, \tilde{G}\right) \subset \operatorname{Hom}\left(\tilde{\Gamma} \star F_{k}, \tilde{G}\right)
$$

as the images of

$$
\operatorname{Hom}_{o}(\Gamma, G) \times G^{k}, \quad \operatorname{Hom}_{o}(\tilde{\Gamma}, \tilde{G}) \times \tilde{G}^{k}
$$

respectively.

It follows from Lemmata 3.13 and 3.14 that $\operatorname{Hom}_{c}(\Gamma, G) \times G^{k}$ is a cross-section for the action of $G$ on $\operatorname{Hom}_{o}(\Gamma, G) \times G^{k}$, while $\operatorname{Hom}_{c}(\tilde{\Gamma}, \tilde{G}) \times \tilde{G}^{k}$ is a cross-section for the action of $G$ on $\operatorname{Hom}_{o}(\tilde{\Gamma}, \tilde{G}) \times \tilde{G}^{k}$. We, thus, obtain:

\section{Lemma 3.16.}

$$
\left(\operatorname{Hom}_{o}(\Gamma, G) \times G^{k}\right) / G \cong X_{o}(\Gamma, G) \times G^{k}
$$

The etale covering $q$ defined above yields, for each $k$, the etale covering

$$
q_{k}: \operatorname{Hom}_{o}(\tilde{\Gamma}, \tilde{G}) \times \tilde{G}^{k} \cong \operatorname{Hom}_{o}\left(\tilde{\Gamma} \star F_{k}, \tilde{G}\right) \rightarrow \operatorname{Hom}_{o}\left(\Gamma \star F_{k}, G\right) \cong \operatorname{Hom}_{o}(\Gamma, G) \times G^{k}
$$

Corollary 3.17. 1. $X_{o}\left(\Gamma \star F_{k}, G\right) \cong X_{o}(\Gamma, G) \times G^{k}$.

2. $\operatorname{Hom}_{o}\left(\tilde{\Gamma} \star F_{k}, \tilde{G}\right) \cong X_{o}(\tilde{\Gamma}, \tilde{G}) \times \tilde{G}^{k}$.

3. The covering $q_{k}$ is étale.

\section{Universality theorem of Panov and Petrunin}

Proof of Theorem 1.1 and its corollaries hinges upon two results, the first of which is the following:

Theorem 4.1 (Panov-Petrunin Universality Theorem, [12]). Let $\Gamma$ be a finitely-presented group. Then there exists a closed 3-dimensional (non-orientable) hyperbolic orbifold $O$ so that $\pi_{1}(Y) \cong \Gamma$, where $Y$ is the underlying space of $O$. Furthermore, $Y$ is a 3-dimensional pseudomanifold without boundary.

Remark 4.2. Examination of the proof in [12] shows that the orbifold $O$ admits a hyperbolic manifold cover $\tilde{O} \rightarrow O$ with the deck-transformation group $\mathbb{Z}_{2}^{4}$.

The singular set of the pseudomanifold $Y$ consists of singular points $y_{j}, j=1, \ldots, 2 k$, whose neighborhoods $C_{j}$ in $Y$ are cones over $\mathbb{R P}^{2}$. Note that, since $\mathbb{R P}^{2}$ has Euler characteristic 1 , the number of conical singularities has to be even. Observe also that one needs $k \geq 1$ in this theorem, since fundamental groups of 3-dimensional manifolds are very restricted among finitely-presented groups. For instance, there are no 3 -manifolds $M$ with $\pi_{1}(M) \cong \mathbb{Z}^{4}$; therefore, for $\Gamma \cong \mathbb{Z}^{4}$, one cannot have $k=0$ in Theorem 4.1. 
Problem 4.3. Does Theorem 4.1 hold with $k=1$ ?

Given $\Gamma$ and $Y$ as in Theorem 4.1, we will construct a closed (non-orientable) 3dimensional manifold $M=M_{\Gamma}$ as follows. (Formally speaking, this 3-manifold also depends on the choice of an orbifold $O$ in Theorem 4.1, which is very far from being unique, however, in order to simplify the notation, we will suppress this dependence).

Let $O$ be a 3-dimensional orbifold as in Theorem 4.1 and let $Y$ be the underlying space of $O$. Let $Y^{\prime}$ be obtained by removing open cones $C_{j}, j=1, \ldots, 2 k$, from $Y$. Then $Y^{\prime}$ is a compact 3 -dimensional manifold with $2 k$ boundary components each of which is a copy of the projective plane $\mathbb{R P}^{2}$. We let $\theta_{i}$ denote the generator of the fundamental group of the projective plane $P_{i} \cong \mathbb{R P}^{2} \subset \partial M$, which equals the boundary of the cone $C_{i}$. We will regard $\theta_{i}$ as an element of $\pi_{1}\left(Y^{\prime}\right)$. Set

$$
\Theta:=\left\{\theta_{1}, \ldots, \theta_{k}\right\}
$$

Then

$$
\Gamma=\pi_{1}(Y)=\pi_{1}\left(Y^{\prime}\right) /\left\langle\left\langle\theta_{1}, \ldots, \theta_{2 k}\right\rangle\right\rangle .
$$

Next, let $M$ be the closed 3-dimensional manifold obtained by attaching $k$ copies of the product $\mathbb{R P}^{2} \times[0,1]$ to $Y^{\prime}$ along the boundary projective planes, pairing the projective planes $P_{i}$ and $P_{i+k}, i=1, \ldots, k$. Then $\pi=\pi_{1}\left(M_{\Gamma}\right)$ is the iterated HNN extension of $\pi_{1}\left(Y^{\prime}\right)$ with stable letters $t_{1}, \ldots, t_{k}$ :

$$
\left(\left(\left(\pi_{1}\left(Y^{\prime}\right) \star\left\langle\theta_{1}\right\rangle\right) \star\left\langle\theta_{2}\right\rangle\right) \ldots\right) \star\left\langle\theta_{k}\right\rangle .
$$

Taking the quotient

$$
\phi: \pi \rightarrow \pi /\langle\langle\Theta\rangle\rangle,
$$

we, therefore, obtain the group $\Gamma \star F_{k}$, where $F_{k}$ is the free group on $k$ generators, projections of the stable letters $t_{i}, i=1, \ldots, k$ in the above HNN extension. We let

$$
\psi: \Gamma \star F_{k} \rightarrow \Gamma
$$

denote the further projection to the first direct factor and set

$$
\xi:=\psi \circ \phi: \pi \rightarrow \Gamma \text {. }
$$

Now, given an algebraic group $H$, we obtain

$$
\operatorname{Hom}_{\Theta}(\pi, H)=\phi^{*}\left(\operatorname{Hom}\left(\Gamma \star F_{k}, H\right)\right),
$$

a clopen subscheme in $\operatorname{Hom}(\pi, H)$ (see Corollary 2.4). The isomorphism

$$
H^{k} \times \operatorname{Hom}(\Gamma, H) \stackrel{\cong}{\longrightarrow} \operatorname{Hom}\left(\Gamma \star F_{k}, H\right) \stackrel{\phi^{*}}{\longrightarrow} \operatorname{Hom}_{\Theta}(\pi, H)
$$

restricted to $1 \times \operatorname{Hom}(\Gamma, H)$ equals $\xi^{*}$. We, thus, we obtain:

Lemma 4.4. For each open subscheme $S \subset \operatorname{Hom}(\Gamma, H)$, there exists an open subscheme $\widehat{S} \subset \operatorname{Hom}(\pi, H)$ isomorphic to $H^{k} \times S$ via the morphism $\phi^{*}$. Furthermore, $\widehat{S}$ contains $\xi^{*}(S)$.

Proof. Take $\widehat{S}=\phi^{*}\left(H^{k} \times S\right)$, where we identify $H^{k} \times \operatorname{Hom}(\Gamma, H)$ with the representation scheme $\operatorname{Hom}\left(\Gamma \star F_{k}, H\right)$. 


\section{A universality theOrem For Coxeter groups}

The second key ingredient we need is the following theorem which is essentially contained in [5]. Before stating the theorem we recall (cf. Lemma 3.13) that the action $G \curvearrowright \operatorname{Hom}_{o}(\Gamma, G)$ has a cross-section $\operatorname{Hom}_{c}(\Gamma, G) \subset \operatorname{Hom}_{o}(\Gamma, G)$, i.e., $\operatorname{Hom}_{o}(\Gamma, G)$ is $G$-equivariantly isomorphic to the product $X_{o}(\Gamma, G) \times G$. As always, $G=P O(3)$.

Theorem 5.1 (M. Kapovich, J. J. Millson). Let $X$ and $x \in X$ be as in Theorem 1.1. Then there exists an open subscheme $X^{\prime} \subset X$ containing $x$, a finitely-generated Coxeter group $\Gamma$ (so that every edge of its graph $\Delta$ has label 2 or 4 ) and a representation $\rho_{c}: \Gamma \rightarrow P O(3, \mathbb{R})$ with dense image, so that $X^{\prime}$ is isomorphic to an open subscheme $S^{\prime} \subset X_{o}(\Gamma, G)$. The representation $\rho_{c}$ belongs to $\operatorname{Hom}_{o}(\Gamma, P O(3, \mathbb{R}))$. Furthermore, under this isomorphism, $x$ corresponds to $\left[\rho_{c}\right]$.

Remark 5.2. The fact that the $\operatorname{Hom}_{o}(\Gamma, G) \cong X_{o}(\Gamma, G) \times G$ (with $\operatorname{Hom}_{c}(\Gamma, G)$ containing $\rho_{c}$ serving as a cross-section for the action $\left.G \curvearrowright \operatorname{Hom}_{o}(\Gamma, G)\right)$ implies that the preimage $R_{o}^{\prime}$ of $S^{\prime}$ in $\operatorname{Hom}_{o}(\Gamma, G)$ is isomorphic to $G \times S^{\prime}=G \times S_{c}^{\prime}$. As we saw in $\$ 2.2$, the representation $\rho_{c}$ lifts to a representation

$$
\tilde{\rho}_{c}: \tilde{\Gamma} \rightarrow S U(2)
$$

of the canonical central extension $\tilde{\Gamma}$ of $\Gamma$.

Since the universality theorems proven in [5] are somewhat different from the one stated above, we outline the proof of Theorem [5.1. The main differences are that the results of [5] are about representations of Shephard and Artin groups rather than Coxeter groups. Furthermore, the representation to $P O(3, \mathbb{R})$ constructed in [5] has finite image (which was important for [5]), although the image group does have trivial centralizer in $P O(3, \mathbb{C})$.

The arguments below are minor modifications of the ones in [5].

Step 1 (Scheme-theoretic version of Mnëv Universality Theorem). Without loss of generality, we may assume that the rational point $x$ is the origin 0 in the affine space containing $X$. In [5] we first construct a based projective arrangement $A$, so that an open subscheme $B R_{0}\left(A, \mathbb{P}^{2}\right)$ in the space of based projective realizations $B R\left(A, \mathbb{P}^{2}\right)$, is isomorphic to $X$ as a scheme over $\mathbb{Q}$, and, moreover, the geometrization isomorphism

$$
X \stackrel{\text { geo }}{\longrightarrow} B R_{0}\left(A, \mathbb{P}^{2}\right)
$$

sends $x \in X$ to a based realization $\psi_{0}: A \rightarrow \mathbb{P}^{2}$ whose image is the standard triangle. Furthermore, the images of the points and lines in $A$ under $\psi_{0}$ are real.

Remark 5.3. Subsequently, a proof of this result was also given by Lafforgue in [8], who was apparently unaware of [5].

Step 2. An arrangement $A$ is a certain bipartite graph containing a subgraph $T$ (the "base") which is isomorphic to the incidence graph of the "standard triangle" (also known as "standard quadrangle"), see [5, Figure 7]. The subgraph $T$ has 5 vertices $v_{00}, v_{x}, v_{y}, c_{10}, v_{01}, v_{11}$ corresponding to the "points" of the standard triangle and 6 vertices $l_{x}, l_{d}, l_{y}, l_{x 1}, l_{y 1}, l_{\infty}$ which correspond to the "lines" of the standard 
triangle. In [5, §11] we further modify the bipartite graph $A$ : We make the following identification of vertices:

$$
v_{00} \sim l_{\infty}, \quad v_{x} \sim l_{y}, \quad v_{y} \sim l_{x}
$$

We also add to $A$ the edges:

$$
\left[v_{10}, v_{00}\right], \quad\left[v_{01}, v_{00}\right] .
$$

We will use the upper case notation $V_{00}=\psi_{0}\left(v_{00}\right), V_{x}=\psi_{0}\left(v_{x}\right)$, etc., to denote vectors in $\mathbb{C}^{3}$ which project to the images under $\psi_{0}$ of the point-vertices of $T$. The choice of this vectors is not unique, of course; we assume that $V_{00}, V_{x}, V_{y}$ form a basis and

$$
V_{10}=V_{00}+V_{x}, \quad V_{01}=V_{00}+V_{y}, \quad V_{11}=V_{00}+V_{x}+V_{y} .
$$

This is possible due to the incidencies in $\psi_{0}(T)$.

However, here, unlike [5], we will not add the edge $\left[v_{00}, v_{11}\right]$. (The purpose of this edge in [5] was to ensure that certain representation of a Shephard group is finite.) We let $A^{\prime}$ denote the resulting graph (no longer bipartite). We assign labels to the edges of $A^{\prime}$ as follows: All edges are labelled 2 except for the two edges

$$
\left[v_{10}, v_{00}\right], \quad\left[v_{01}, v_{00}\right],
$$

which have the label 4 . We then let $\Gamma$ denote the Coxeter group corresponding to this labelled graph. We let $T^{\prime}$ denote the labelled subgraph of $A^{\prime}$, whose vertices are the images of the vertices of the arrangement $T$.

The labeled graph $\Omega$ as in Fugure 1 embeds into $T^{\prime}$ via the map

$$
v \mapsto v_{00}, \quad x \mapsto v_{10}, \quad y \mapsto v_{01}, \quad u \mapsto v_{x}, \quad w \mapsto v_{y}
$$

We equip the vector space $\mathbb{C}^{3}$ with a nondegenerate bilinear form, so that:

1. All subspaces which appear in the image $\psi_{0}(T)=\psi_{0}(A)$ are anisotropic (the bilinear form has nondegenerate restriction to these subspaces).

2. The vectors $V_{00}, V_{x}, V_{y} \in \mathbb{C}^{3}$ are pairwise orthogonal and have unit norm.

We let $P O(3)$ denote the projectivization of the orthogonal group $O(3)$ preserving this bilinear form.

A realization $\psi \in R\left(A, \mathbb{P}^{2}\right)$ is anisatropic if for each vertex $v \in A$, the image $\psi(v)$ is an anisatropic subspace in $\mathbb{C}^{3}$. We will use the notation $R_{a}\left(A, \mathbb{P}^{2}\right) \subset R\left(A, \mathbb{P}^{2}\right)$ and $B R_{a}\left(A, \mathbb{P}^{2}\right) \subset B R\left(A, \mathbb{P}^{2}\right)$ for open schemes of anisotripic realizations and anisotropic based realizations. By the condition (1) on the inner product above, $B R_{a}\left(A, \mathbb{P}^{2}\right)$ containins $\psi_{0}$.

To every anisotropic realization $\psi \in R\left(A, \mathbb{P}^{2}\right)$, we associate a representation of the group $\Gamma$ by sending every generator $g_{v} \in \Gamma$ to the isometric involution in $P O(3)$ fixing the subspace $\psi(v)$ in $\mathbb{P}^{2}$. As in [5], this map of generators of $\Gamma$ to $P O(3)$ defines a representation

$$
\rho_{\psi}: \Gamma \rightarrow P O(3, \mathbb{C})
$$

We define

$$
\rho_{c}:=\rho_{\psi_{0}} .
$$

By the construction, each representation $\rho_{\psi}$ is faithful on elementary subgroups: For the edges $[v, w]$ in $A$ (where $v$ is a point and $w$ is a line), the incidence condition 
$\psi(v) \in \psi(v)$ in $P^{2}$ forces the point reflection in $\psi(v)$ be distinct from the line reflection in $\psi(w)$. For the edges

$$
\left[v_{10}, v_{00}\right], \quad\left[v_{01}, v_{00}\right]
$$

the condition (6) forces the point reflections in $\psi\left(v_{00}\right), \psi\left(v_{10}\right), \psi\left(v_{01}\right)$ to be pairwise noncommutting and, hence, both subgroups

$$
\begin{aligned}
& \rho_{\psi}\left(\left\langle g_{v_{00}}, g_{v_{10}}\right\rangle\right)<P O(3, \mathbb{C}) \\
& \rho_{\psi}\left(\left\langle g_{v_{00}}, g_{v_{01}}\right\rangle\right)<P O(3, \mathbb{C})
\end{aligned}
$$

are isomorphic to $I_{2}(4)$. We also note that

$$
\left.\rho_{\psi}\right|_{\Gamma_{\Omega}}=\rho_{\Omega}:=\rho_{\psi_{0}}: \Gamma_{\Omega} \rightarrow \operatorname{PO}(3, \mathbb{C}) .
$$

We, thus, obtain the algebraization morphism of schemes

$$
\text { alg }: B R_{a}\left(A, \mathbb{P}^{2}\right) \rightarrow \operatorname{Hom}(\Gamma, P O(3)), \quad \psi \mapsto \rho_{\psi} .
$$

As in [5], the morphism alg is an isomorphism to its image. It follows from Lemma 3.13 and (7) that the subscheme

$$
S_{c}:=\operatorname{alg}\left(B R_{a}\left(A, \mathbb{P}^{2}\right)\right) \subset \operatorname{Hom}_{c}(\Gamma, P O(3)) \subset \operatorname{Hom}_{o}(\Gamma, P O(3))
$$

is a cross-section for the action of $G$ on the $G$-orbit of $S_{c}$.

Let $\Sigma \subset A^{\prime}$ denote the complete subgraph whose vertices are the vertices (points and lines) of the standard triangle in $A$, except for the vertex $v_{11}$. As in [5], the image under $\rho_{c}$ of the corresponding parabolic Coxeter subgroup $\Gamma_{\Sigma} \subset \Gamma$, is isomorphic to the finite Coxeter group $B_{3}$ (the symmetry group of the regular octahedron) divided by the center $\mathbb{Z}_{2}$. Such a group is a maximal finite subgroup of $P O(3, \mathbb{R})$. However, the involution $\rho_{c}\left(g_{v_{11}}\right)$ does not belong to the group $\rho_{c}\left(\Gamma_{\Sigma}\right)$ (this would be order 2 rotation in the center of a face of the octahedron). Thus, the group $\rho_{c}(\Gamma)$ has to be dense in $P O(3, \mathbb{R})$, as it contains (actually, equals to) the dense subgroup $\rho_{c}\left(\Gamma_{T^{\prime}}\right)$. This is the only essential difference between the construction in this paper and in [5], where it was important for the group $\rho_{c}(\Gamma)$ to be finite.

We let

$$
\mu: \operatorname{Hom}_{o}(\Gamma, G) \rightarrow X_{o}(\Gamma, G) .
$$

denote the restriction of the GIT quotient $\operatorname{Hom}(\Gamma, G) \rightarrow X(\Gamma, G)$. Since $\operatorname{Hom}_{c}(\Gamma, G)$ is a cross-section for the $G$-action on $\operatorname{Hom}_{o}(\Gamma, G)$, the morphism $\mu$ is a trivial principal G-bundle.

Theorem 5.4. alg: $B R_{a}\left(A, \mathbb{P}^{2}\right) \rightarrow \operatorname{Hom}_{c}(\Gamma, P O(3, \mathbb{C}))$ is an isomorphism.

Proof. We will only sketch the proof since it follows closely the argument in Theorem 12.14 in [5] and the latter is quite long. One verifies that alg induces a natural isomorphism of functors of points. For instance, over the complex numbers, each representation $\rho \in \operatorname{Hom}_{c}(\Gamma, P O(3, \mathbb{C}))$ gives rise to an anisotropic realization: $\psi(v) \in$ $\mathbb{P}^{2}(\mathbb{C})$ is the point fixed by $\rho\left(g_{v}\right)$ (if $v$ is a point-vertex) and $\psi(v) \in \mathbb{P}^{2}(\mathbb{C})$ is the line fixed by $\rho\left(g_{v}\right)$ (if $v$ is a line-vertex).

Corollary 5.5. 1. $S_{c}$ is a cross-section for the action of $G$ on $\operatorname{Hom}_{o}(\Gamma, G)$.

2. $\mu \circ$ alg: $B R_{a}\left(A, \mathbb{P}^{2}\right) \rightarrow X_{o}(\Gamma, G)$ is an isomorphism.

3. $S:=\mu \circ \operatorname{alg}\left(B R_{a}\left(A, \mathbb{P}^{2}\right)\right) \subset X(\Gamma, G)$ is an open subscheme. 
Proof. Part 1 follows from the fact that

$$
S_{c}=\operatorname{alg}\left(B R_{a}\left(A, \mathbb{P}^{2}(\mathbb{C})\right)=\operatorname{Hom}_{c}(\Gamma, P O(3, \mathbb{C}))\right.
$$

and the latter is a cross-section for the $G$-action on $\operatorname{Hom}_{o}(\Gamma, G)($ Lemma 3.13). Part 2 is immediate from Theorem 5.4 and Part 1. Part 3 follows from the fact that $X_{o}(\Gamma, G)$ is an open subscheme in $X(\Gamma, G)$.

We define $X^{\prime}:=g e o^{-1}\left(B R_{a}\left(A, \mathbb{P}^{2}\right)\right) \subset X$, an open subscheme in $X$. The composition of geo, alg and $\mu$, yields an isomorphism $\kappa: X^{\prime} \rightarrow S^{\prime}$,

$$
\begin{aligned}
& X \supset X^{\prime} \stackrel{\text { geo }}{\longrightarrow} B R_{a}\left(A, \mathbb{P}^{2}\right) \cap B R_{0}\left(A, \mathbb{P}^{2}\right) \stackrel{\text { alg }}{\longrightarrow} S_{c} \stackrel{\mu}{\longrightarrow} S \subset X_{o}(\Gamma, G), \\
& \kappa\left(X^{\prime}\right)=S^{\prime} \subset S,
\end{aligned}
$$

where $X^{\prime} \subset X$ and $S^{\prime} \subset S \subset X_{o}(\Gamma, G)$ are open subsechemes, The isomorphism $\kappa$ sends the point $x \in X^{\prime}$ to $\left[\rho_{c}\right] \in X_{o}(\Gamma, G)$. This concludes the proof of Theorem 5.1 ,

We let $R_{o}^{\prime}$ denote the preimage of $S^{\prime}$ in $\operatorname{Hom}_{o}(\Gamma, G)$ and define

$$
S_{c}^{\prime}=S_{c} \cap R_{o}^{\prime} \text {. }
$$

Then

$$
R_{o}^{\prime}=G \cdot S_{c}^{\prime} \cong G \times S_{c}^{\prime} .
$$

By inverting the isomorphism $\kappa$ and multiplying with the identity map $G \rightarrow G$, we obtain:

Corollary 5.6. There exists an isomorphism of schemes over $\mathbb{Q}$

$$
\omega: R_{o}^{\prime} \rightarrow G \times X^{\prime}
$$

\section{Proof of Theorem 1.1}

We continue with notation introduced in the previous sections. Given an affine scheme $X$ over $\mathbb{Q}$ and a rational point $x \in X$, we use Theorem 5.1 to construct a Coxeter group $\Gamma$ and a representation $\rho_{c}: \Gamma \rightarrow P O(3, \mathbb{R})<P O(3, \mathbb{C})$. Then, as in $\S 4$, we will construct a closed 3-manifold $M=M_{\Gamma}$ with the fundamental group $\pi$, and a clopen subscheme $\operatorname{Hom}_{o}(\pi, G)$ which is isomorphic to the product $\operatorname{Hom}_{o}(\Gamma, G) \times G^{k}$. In (5) we defined an epimorphism

$$
\xi: \pi \stackrel{\phi}{\longrightarrow} \Gamma \star F_{k} \stackrel{\psi}{\longrightarrow} \Gamma .
$$

Define $\rho_{0}=\xi^{*}\left(\rho_{c}\right) \in \operatorname{Hom}(\pi, G)$. The subgroup $\rho_{0}(\pi)=\rho_{c}(\Gamma)<G(\mathbb{R})$ is dense according to Theorem 5.1 .

We next "convert" the open subscheme $R_{o}^{\prime} \subset \operatorname{Hom}_{o}(\Gamma, G)$ to an open subscheme $R^{\prime} \subset \operatorname{Hom}(\pi, G)$. The obvious choice $\xi^{*}\left(R_{o}^{\prime}\right)$ will not be open in $\operatorname{Hom}(\pi, G)$. Instead, we use the open subscheme $R^{\prime}:=\widehat{R_{o}^{\prime}} \subset \operatorname{Hom}(\pi, G)$ defined in Lemma 4.4.

By combining the isomorphism

$$
R^{\prime} \rightarrow G^{k} \times R_{o}^{\prime} \subset G^{k} \times \operatorname{Hom}(\Gamma, G)
$$

with the isomorphism

$$
i d \times \omega: G^{k} \times R_{o}^{\prime} \rightarrow G^{k} \times\left(G \times X^{\prime}\right)
$$


(where $\omega$ is from Corollary [5.6), we obtain an isomorphism

$$
f: R^{\prime} \stackrel{\cong}{\longrightarrow} G^{k} \times R_{o}^{\prime} \stackrel{\cong}{\longrightarrow} G^{k+1} \times X^{\prime} \subset G^{k+1} \times X,
$$

sending $\rho_{0} \in R^{\prime}$ to

$$
x^{\prime}=1 \times x \in G^{k+1} \times X^{\prime} .
$$

By the construction, $R^{\prime}$ is open in $\operatorname{Hom}(\pi, G)$ and $G^{k+1} \times X^{\prime}$ is open in $G^{k+1} \times X$.

The cross-section $S_{c}^{\prime} \subset H_{o m}(\Gamma, G)$ in Theorem 5.1 yields a cross-section $R_{c}^{\prime} \subset R \subset$ $\operatorname{Hom}(\pi, G)$ for the action $G \curvearrowright R^{\prime}$ :

$$
R_{c}^{\prime}=\phi^{*}\left(G^{k} \times \psi^{*}\left(S_{c}^{\prime}\right)\right) .
$$

This concludes the proof of Theorem 1.1.

\section{Corollaries of Theorem 1.1}

Theorem 1.1 deals with representation schemes of 3-manifold groups to $G=P O(3)$; we now consider the corresponding character schemes. Since $R_{c}^{\prime} \subset \operatorname{Hom}_{o}(\pi, G)$ is a cross-section for the action of $G$ on $R^{\prime}$, Theorem 1.1 immediately implies:

Corollary 7.1. With the notation of Theorem 1.1, there exists an open embedding of schemes:

$$
G^{k} \times X^{\prime} \hookrightarrow X_{o}(\pi, G)=H_{o m}(\pi, G) / / G
$$

which sends $(1, x)$ to $\left[\rho_{0}\right]$. In particular, the analytic germ $\left(\mathbb{C}^{3 k} \times X^{\prime}, 0 \times x\right)$ is isomorphic to the analytic germ $\left(X(\pi, G),\left[\rho_{0}\right]\right)$.

We next consider representations of 3-manifold groups to the group $\tilde{G}=S L(2)$; we work over $\mathbb{C}$ and, thus, identify $P S L(2, \mathbb{C})$ with $P O(3, \mathbb{C})$. Recall that, according to Theorem 5.1 (and Remark 5.2), for every affine scheme $X$ over $\mathbb{Q}$ and a rational point $x \in X$, there exists an open subscheme $X^{\prime} \subset X$ containing $x$, a Coxeter group $\Gamma$ an open subscheme $G \times S_{c}^{\prime} \cong R_{o}^{\prime} \subset \operatorname{Hom}(\Gamma, G)$, and an isomorphism of schemes over $\mathbb{C}$ (which is the identity on the $G$-factor):

$$
R_{o}^{\prime}=G \times S_{c}^{\prime} \cong G \times S^{\prime} \rightarrow G \times X^{\prime}
$$

sending $\rho_{c} \in S_{c}^{\prime} \subset \operatorname{Hom}_{c}(\Gamma, G) \subset \operatorname{Hom}_{o}(\Gamma, G) \subset \operatorname{Hom}(\Gamma, G)$ to $1 \times x$.

Now, consider representations of the corresponding extended Coxeter group $\tilde{\Gamma}$. Proposition 3.8 gives us a $G$-equivariant regular étale covering

$$
q: \operatorname{Hom}_{o}(\tilde{\Gamma}, \tilde{G}) \rightarrow \operatorname{Hom}_{o}(\Gamma, G)
$$

with the covering group $\mathbb{Z}_{2}^{r}$. Restricting to $R_{o}^{\prime} \subset \operatorname{Hom}_{o}(\Gamma, G)$ we obtain a $G$-equivariant regular etale covering

$$
q^{\prime}: \tilde{R}_{o}^{\prime} \rightarrow R_{o}^{\prime}, \quad \tilde{R}_{o}^{\prime}=q^{-1}\left(R_{o}^{\prime}\right) \subset \operatorname{Hom}_{o}(\tilde{\Gamma}, \tilde{G}), \quad q^{\prime}=\left.q\right|_{\tilde{R}_{o}^{\prime}} .
$$

We let $\tilde{\rho}_{c}: \tilde{\Gamma} \rightarrow \tilde{G}(\mathbb{C})$ be a lift of $\rho_{c}$. The subscheme $S=\tilde{R}_{o}^{\prime}$ is open in $\operatorname{Hom}(\tilde{\Gamma}, \tilde{G})$.

We now repeat the proof of Theorem 1.1. Given the group $\tilde{\Gamma}$ we construct a closed 3 -manifold with the fundamental group $\tilde{\pi}$ which admits an epimorphism

$$
\tilde{\xi}: \tilde{\pi} \rightarrow \tilde{\Gamma} \times F_{k} \rightarrow \tilde{\Gamma} .
$$

Set $\tilde{\rho}_{0}:=\xi^{*}\left(\tilde{\rho}_{c}\right)$. 
Lemma 4.4 (applied to $S$ ) yields an open subscheme $\widehat{S} \subset \operatorname{Hom}(\tilde{\pi}, \tilde{G})$ isomorphic to $\tilde{G}^{k} \times \tilde{R}_{o}^{\prime}$. Combining this isomorphism with the etale covering

$$
\tilde{G}^{k} \times \tilde{R}_{o}^{\prime} \rightarrow \tilde{G}^{k} \times R_{o}^{\prime}
$$

and the isomorphism

$$
R_{o}^{\prime} \cong G \times X^{\prime}
$$

we obtain a regular etale covering

$$
\tilde{f}: \widehat{S} \rightarrow G^{k+1} \times X^{\prime},
$$

sending the representation $\tilde{\rho}$ to $1 \times x$. The group of covering transformations of $\tilde{f}$ is the group $\mathbb{Z}_{2}^{r}$ (coming from the covering $\tilde{R}_{o}^{\prime} \rightarrow R_{o}^{\prime}$ ).

Furthermore, the subgroup $\tilde{\rho}_{0}(\pi) \subset S L(2, \mathbb{C})$ is Zariski dense over $\mathbb{C}$, since this subgroup is dense in $S U(2)$ (because it projects to a dense subgroup $\rho_{c}(\Gamma) \subset P O(3, \mathbb{R})$ ). Since all groups $p(\tilde{\rho}(\pi)), \tilde{\rho} \in \operatorname{Hom}_{o}(\pi, \tilde{G})$, contain a conjugate of the group

$$
\rho_{c}\left(\Gamma_{T^{\prime}}\right),
$$

it also follows that for every $\tilde{\rho} \in \widehat{S}$, the group $\tilde{\rho}(\pi)$ is Zariski dense in $S L(2, \mathbb{C})$. (The subgraph $T^{\prime} \subset A^{\prime}$ is defined in $\$ 5$ ); the group $\Gamma_{T^{\prime}} \subset \Gamma_{A^{\prime}}=\Gamma$ is the corresponding parabolic subgroup.) This proves Corollary 1.3.

\section{ORBIFOLD-GROUP REPRESENTATIONS}

Let $\hat{\Gamma}$ be the fundamental group of the hyperbolic orbifold appearing in Theorem 4.1. This group contains cyclic subgroups $\left\langle\theta_{i}\right\rangle \cong \mathbb{Z}_{2}, i=1, \ldots, 2 k$, corresponding to the singular points $y_{i}$. The group $\Gamma$ is the quotient

$$
\hat{\Gamma} /\langle\langle\hat{\Theta}\rangle\rangle
$$

where $\hat{\Theta}=\left\{\theta_{1}, \ldots, \theta_{2 k}\right\} \subset \hat{\Gamma}$. Then for every algebraic group $H$,

$$
\operatorname{Hom}(\Gamma, H) \cong \operatorname{Hom}_{\hat{\Theta}}(\hat{\Gamma}, H)
$$

and the latter is an open subscheme in $\operatorname{Hom}(\hat{\Gamma}, H)$ (see Corollary 2.4). Now, let $\Gamma$ be a Coxeter group (as in Theorem 5.11) or its canonical central extension. In view of Theorems 4.1 and 5.1, one obtains:

Corollary 8.1. Theorems 1.1 and Corollaries 1.3, 1.5, also hold for groups $\pi$ which are fundamental groups of 3-dimensional closed hyperbolic orbifolds.

By passing to a finite-index torsion-free subgroups of $\pi$, in view of [4, Theorem 5.1], we obtain new examples of fundamental groups of hyperbolic 3-manifolds and their representations to $S O(3), S U(2)$ with non-quadratic singularities of character varieties, cf. [4, Theorem 5.1] where it is proven that nonquadratic singularities of character schemes are inherited by finite index subgroups. (The first such examples were constructed in [4.)

Question 8.2. Do Theorems 1.1 and Corollaries 1.3, 1.5, also hold for groups $\pi$ which are fundamental groups of 3-dimensional closed hyperbolic manifolds? Do they hold for 3-dimensional manifolds which are 3-dimensional (integer or rational) homology spheres? 
Older examples. We note that the first example of a nonreduced representation scheme was constructed by Lubotzky and Magid in [9, p. 43]: They start with the von Dyck group

$$
\Gamma=\left\langle a, b, c \mid a^{3}=b^{3}=c^{3}=a b c=1\right\rangle
$$

and its representation

$$
\rho: \Gamma \rightarrow S L(2, \mathbb{C})
$$

whose image is a cyclic group $\mathbb{Z}_{3}$ of order 3 ( $\rho$ sends $a, b$ and $c$ to the same generator of $\left.\mathbb{Z}_{3}\right)$. Then $\mathrm{H}^{1}(\Gamma, A d \rho) \cong \mathbb{C}$, while $[\rho] \in X^{\text {red }}(\Gamma, S L(2, \mathbb{C}))$ is an isolated point. It follows that $B^{1}(\Gamma, A d \rho) \cong \mathbb{C}^{2}$ and, therefore,

$$
Z^{1}(\Gamma, A d \rho) \cong \mathbb{C}^{3}
$$

The same, of course, holds for all representations $\Gamma \rightarrow S L(2, \mathbb{C})$ conjugate to $\rho$. On the other hand, the component $C$ of $\rho$ in $\operatorname{Hom}^{\text {red }}(\Gamma, S L(2, \mathbb{C}))$ is a smooth 2-fold, isomorphic to $S L(2, \mathbb{C}) / T$, where $T \cong \mathbb{C}^{*}$ is a maximal torus in $S L(2, \mathbb{C})$. In contrast, the Zariski tangent space $T_{c} C$ at each $c \in C$ is isomorphic to $Z^{1}(\Gamma, A d \rho) \cong \mathbb{C}^{3}$. It follows that the representation scheme $\operatorname{Hom}(\Gamma, S L(2))$ is nonreduced.

This example is promoted to a nonreduced representation scheme of a 3-manifold group as follows. Consider a closed 3-dimensional Seifert manifold $M$ which is an oriented Seifert-bundle over the orbifold $S^{2}(3,3,3)$, i.e., over the sphere with 3 cone points of order 3. The fundamental group of $S^{2}(3,3,3)$ is the von Dyck group $\Gamma$ above, while $\pi=\pi_{1}(M)$ is the central extension of $\Gamma$ :

$$
\begin{gathered}
1 \rightarrow \mathbb{Z} \rightarrow \pi \rightarrow \Gamma \rightarrow 1 \\
\pi=\left\langle a, b, c, z \mid a^{3}=b^{3}=c^{3}=a b c=z,[a, z]=[b, z]=[c, z]=1\right\rangle .
\end{gathered}
$$

The representation $\rho$ lifts to a representation $\tilde{\rho}: \pi \rightarrow S L(2, \mathbb{C})$ whose kernel contains the center $\langle z\rangle$ of $\pi$. Then, a direct computation shows that

$$
\mathrm{H}^{1}(\pi, A d \rho) \cong \mathbb{C}^{2}, \quad Z^{1}(\pi, A d \rho) \cong \mathbb{C}^{4},
$$

while the germ of the reduced representation variety $\operatorname{Hom}^{\text {red }}(\pi, S L(2, \mathbb{C}))$ at $\tilde{\rho}$ is a smooth 3 -fold, consisting of reducible representations $\pi \rightarrow S L(2, \mathbb{C})$. Up to conjugation, these representations all have the form

$$
\rho_{t}(a)=\rho_{t}(b)=\rho_{t}(c)=\left[\begin{array}{cc}
t & 0 \\
0 & t^{-1}
\end{array}\right], \quad t \in \mathbb{C}^{*} .
$$

The advantage of the examples constructed in Theorem 1.1 and its corollaries, is that the representation and character schemes constructed there are nonreduced at points corresponding to representations with trivial centralizer.

\section{REFERENCES}

[1] W. Goldman and J. J. Millson, The deformation theory of representations of fundamental groups of compact Kähler manifolds, Inst. Hautes Études Sci. Publ. Math. no. 67 (1988) p. 43-96.

[2] J. Huebschmann, Singularities and Poisson geometry of certain representation spaces, In: "Quantization of singular symplectic quotients," p. 119-135, Progr. Math., 198, Birkhäuser, Basel, 2001.

[3] J. Humphreys, "Reflection Groups and Coxeter Groups", Cambridge Studies in Advanced Mathematics, Vol. 29. Cambridge University Press, 1997. 
[4] M. Kapovich, J. J. Millson, On the deformation theory of representations of fundamental groups of hyperbolic 3-manifolds, Topology, Vol. 35, N 4 (1996) p. 1085-1106.

[5] M. Kapovich and J. J. Millson, On representation varieties of Artin groups, projective arrangements and the fundamental groups of smooth complex algebraic varieties, Inst. Hautes Études Sci. Publ. Math. no. 88 (1999) p. 5-95.

[6] M. Kapovich and J. J. Millson, Moduli spaces of linkages and arrangements, In: "Progress in Geometry", J.-L. Brylinski, et al (eds.), Progress in Mathematics, Birkhäuser, Vol. 172 (1999) p. 237-270

[7] M. Kapovich and J. J. Millson, Universality theorems for configuration spaces of planar linkages, Topology, Vol. 41 (2002), no. 6, p. 1051-1107.

[8] L. Lafforgue, "Chirurgie des Grassmanniennes," CRM Monograph Series, Vol. 19, Amer. Math. Soc., 2003.

[9] A. Lubotzky and A. Magid, "Varieties of representations of finitely generated groups," Mem. Amer. Math. Soc. Vol. 58 (1985), no. 336.

[10] D. Johnson, J. J. Millson, Deformation spaces associated to compact hyperbolic manifolds, In: "Discrete groups in geometry and analysis" (New Haven, Conn., 1984), p. 48-106, Progr. in Math., 67, Birkhäuser Boston, Boston, MA, 1987.

[11] N. Mnëv, The universality theorems on the classification problem of configuration varieties and convex polytopes varieties, Springer Lecture Notes in Math., Vol. 1346 (1988) p. 527-543.

[12] D. Panov and A. Petrunin, Telescopic actions, Geometric Analysis and Functional Analysis, Vol. 22 (2012) no. 6, p. 1814-1831.

[13] S. Payne, Moduli of toric vector bundles, Compos. Math. Vol. 144 (2008), no. 5, p. 1199-1213.

[14] I. Rapinchuk, On the character varieties of finitely generated groups, Math. Res. Lett. 22 (2015), no. 2, p. 579-604.

[15] J. Richter-Gerbert, "Realization spaces of polytopes", Springer Lecture Notes in Math., Vol. 1643, (1996).

[16] P. Shalen, Representations of 3-manifold groups, In: "Handbook of geometric topology", p. 955-1044, North-Holland, Amsterdam, 2002.

[17] A. Sikora, Character varieties, Trans. Amer. Math. Soc. Vol. 364 (2012), no. 10, p. 5173-5208.

[18] R. Vakil, Murphy's law in algebraic geometry: Badly-behaved deformation spaces, Inventiones Mathematicae Vol. 164 (2006) p. 569-590.

Addresses:

M.K.: University of California, Davis, CA 95616

kapovich@math.ucdavis.edu

J.M.: University of Maryland, College Park, MD 20742

jjm@math. umd.edu 